\title{
EFAS-Abstracts 2011
}

\section{Free Communications}

\section{Wednesday, 12th October, 2011, 15:00-16:00 h}

\section{FC1}

\section{An autonomic study of the cardiovascular components of yawn}

Camacho-García $B^{1}$, Amores Johanssen $J A^{2}$, Castillo Jimena $M^{2}$, Cuenca Gómez $J^{2}$, Gallego Molina $\mathrm{MB}^{2}$, Vergaz Ballesteros $A^{2}$, Dawid-Milner $M S^{l}$

${ }^{1}$ Unidad de Neurofisiología del Sistema Nervioso Autónomo (CIMES); Universidad de Málaga, Málaga, Spain. ${ }^{2}$ Departamento de Fisiología Humana; Facultad de Medicina; Universidad de Málaga, Málaga, Spain

Yawning is a reflex characterized by a single long lasting deep inspiration, with open mouth and stretching of the eardrums, followed by a shorter exhalation of breath. There are a number of theories that attempt to explain why we yawn. However, the adaptive/functional/ biological significance of yawning has yet to be established. As yawn resembles a deep breathing we have analysed the cardiovascular autonomic components that characterize this response.

Twelve healthy volunteers ( 5 females and 7 males, mean age $22 \pm 1.3$ years) were included in this study. None of these subjects had clinical signs of $\mathrm{CV}$, neurological or metabolic disorders, and none was under medication. Arterial blood pressure (ABP), ECG and respiration were continuously monitored. All participants were video taped during the experiments. Instantaneous heart rate, systolic, diastolic, and mean $\mathrm{ABP}$ changes were analysed during the visualization, in a projection screen, of a 20 min video with images of humans yawing. The test evoked 66 yawns in 7 subjects. After the test, subjects with positive responses, were instructed to produce three false yawns.

Statistical analysis of 66 yawns and 21 false yawns showed no differences in total yawn durations. During the inspiratory phase of true and false yawns an increase in blood pressure $(\mathrm{p}<0.001$ in both groups) and heart rate ( $\mathrm{p}<0.001$ in both groups) was observed. No differences were observed between groups.

During the expiratory phase of yawn the increase in heart rate persisted (98.58 $\pm 1.56 \mathrm{bpm})$. In false yawns an abrupt decrease in heart rate was observed $(85.38 \pm 3.77 \mathrm{bpm})$ thus producing a significant difference between the heart rate components of the expiratory phases of both responses $(p>0.001)$.

These results suggest that during yawn there is an inhibition of the heart rate component of the baroreceptor response, thus increasing sympathetic drive, which keeps a state of arousal that serves to maintain an optimal mental efficiency. The neurophysiological origin of this difference will be discussed.

\section{FC2}

Mid-regional pro-adrenomedullin: a link between nocturnal abnormalities in blood pressure and neurogenic orthostatic hypotension in Parkinson's disease

Despas $F^{l}$, Senard $A^{l}$, Chebane $L^{1}$, Maupas-Schwalm $F^{1}$, Laplace $N^{1}$, Pathak $N^{1}$, Senard $J M^{1}$, Despas $F^{2}$, Senard $A^{2}$, Chebane $L^{2}$, Maupas-Schwalm $F^{2}$, Laplace $N^{2}$, Pathak $A^{2}$, Senard $J^{2}$

${ }^{1}$ University, INSERM, Toulouse, France. Mid-regional proadrenomedullin: a link between nocturnal abnormalities in blood pressure and neurogenic orthostatic hypotension in Parkinson's disease. ${ }^{2}$ Autonomic Unit, Departments of Clinical Pharmacology and Biochemistry, INSERM 1048, University of Toulouse, France

Rationale/objectives: Neurogenic orthostatic hypotension (NOH) is frequently associated with nocturnal abnormalities in blood pressure (NABP) in autonomic failure but the link between both is not understood. The aim of the work was to investigate if adrenomedullin, which is elevated in hypertension and is a potent vasodilator, could be involved in both NABP and $\mathrm{NOH}$.

Methods: 23 patients suffering from Parkinson's disease $(61 \pm$ 2 years, $56.5 \%$ of males, H\&Y stage: $2.1 \pm 0.2$ ) and autonomic failure (at least 2 abnormal responses to classical autonomic testing) were included. Patients with arterial hypertension, obesity, renal failure and diabetes mellitus were excluded. According to the results of a $24 \mathrm{~h}$ blood pressure recording and of $60^{\circ}$ head-up tilt they were classified as normal or suffering from NABP with or without NOH. Plasma MR-pro-AM, a surrogate for adrenomedullin, was measured by fluoroimmunoassays using time resolved amplified cryptate emission (TRACE) technology on the automated system KRYPTOR ${ }^{\circledR}$ analyzer in supine position and at the end of a 10 min head-up tilt. 
Results: NOH was significantly more frequent in patients with NABP ( 78 vs. $20 \%$ in patients without NABP). MR-pro-AM plasma levels were positively correlated with night systolic $\left(\mathrm{r}^{2}: 0.269 ; \mathrm{p}: 0.03\right)$ and diastolic $\left(\mathrm{r}^{2}: 0.307 ; \mathrm{p}: 0.02\right)$ blood pressure and were significantly higher in patients with NABP than in controls $(0.54 \pm 0.03$ vs. $0.39 \pm 0.04 \mathrm{nmol} / \mathrm{L}$, $\mathrm{p}<0.05)$ as well as in patients with $\mathrm{NOH}(0.55 \pm 0.03$ vs. $0.38 \pm 0.04$ $\mathrm{nmol} / \mathrm{L}$ in the absence of $\mathrm{NOH}, \mathrm{p}<0.001)$.

Conclusion: Our data suggest that adrenomedullin plasma levels are elevated in NABP. This could explain the occurrence of NOH is these patients.

\section{FC3}

Pulse wave amplitude variations are modulated by cyclic alternating pattern (CAP) in patients with nocturnal frontal lobe epilepsy

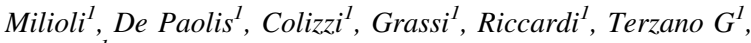 \\ Parrino \\ ${ }^{1}$ University of Parma, Parma, Italy
}

Aim: Sudden drops in pulse wave amplitude (PWA) measured by pulse oximetry result from autonomic vasoconstriction and are associated with simultaneous changes in cortical activity during sleep. The study investigates the relationship between PWA drops and EEG arousal fluctuations expressed during sleep by the cyclic alternating pattern (CAP) in patients with nocturnal frontal lobe epilepsy (NFLE).

Methods: A total of 20 video-polysomnographic recordings carried out in young adults (mean age $25 \pm 7$ years, 12 males) or diagnosis of NFLE were analyzed. Sleep stages and CAP measures were scored according to standardized rules. CAP A phases were detected in all recordings during non-REM sleep and were classified into 3 subtypes (A1, A2, and A3). Only PWA drops $\geq 30 \%$ were included for analysis. CAP was scored by a blind investigator to the PWA signal. The PWA drop was considered associated with a phase A only when the latter occurred $10 \mathrm{~s}$ before or $15 \mathrm{~s}$ after the onset of the PWA decrease.

Results: In NFLE patients CAP rate (76\%) was significantly higher ( $\mathrm{p}<0.0001)$ compared to normative age-balanced values $(32 \%)$. A total of 5,870 PWA drops and 11,234 CAP A phases were scored in non-REM sleep. 4,561 PWA drops $(78 \%)$ were associated with a CAP A phase. The amount of A phases occurring $10 \mathrm{~s}$ before the PWA drops (3004) were significantly more numerous $(\mathrm{p}<0.0001)$ compared to the A phases (359) identified $15 \mathrm{~s}$ after the PWA fall. Subtypes A3 occurring $10 \mathrm{~s}$ before PWA drops were significantly more abundant $(\mathrm{p}<0.0001)$ compared to CAP phases A1 and A2. Conclusions: The high values of CAP rate and the powerful modulation of CAP on PWA variations suggest an altered autonomic balance in NFLE patients. The effects of antiepileptic drugs on the autonomic functions remain to be explored. As a general consideration, drops in PWA highly associated with CAP A phases confirm that PWA recording can be used as a marker of cortical-subcortical activation. These premises can be exploited in cardio-respiratory monitoring of sleep disordered breathing.

\section{FC4}

\section{Video-polysomnographic (V-PSG) findings after cervical and dorsal spinal cord spinal cord injury}

Guaraldi $P^{l}$, Calandra-Buonaura $G^{l}$, Grimaldi $D^{l}$, Cecere $A^{l}$, de Scisciolo $G^{2}$, Aito $S^{2}$, Caramelli $R^{2}$, Schiavone $V^{2}$, Cortelli $P^{1}$ ${ }^{1}$ Department of Neurological Sciences, University of Bologna, Bologna, Italy. ${ }^{2}$ Spinal Unit, AOUC (Azienda Ospedaliera Careggi), Florence, Florence, Italy
Aim: Previous epidemiological studies demonstrated that sleep problems are common in spinal cord injury (SCI) (Jensen et al. 2009), although, so far, only limited polysomnographic data are available (Adey et al. 1968; Scheer et al. 2006). Aim of the study was to assess with a $24 \mathrm{~h}$ video-polysomnography (V-PSG) the sleep pattern of 5 cervical SCI with tetraplegia, 7 thoracic SCI with paraplegia and 14 control subjects.

Methods: Sleep states were visually scored on $30 \mathrm{~s}$ epochs according to the standard Rechtschaffen and Kales criteria as light (stages N1 and N2) non-REM sleep (NREMS), deep (stages N3 and N4) NREMS, and REM sleep. Periodic limbs movement in sleep (PLMS), arousals, PLMS associated with arousal and the corresponding PLMS Index, Arousal Index and PLMS-Arousal Index were scored according to the American Association of Sleep Medicine rules. Nonparametric comparisons were performed by using Mann-Whitney test; a $\mathrm{p}<0.05$ was considered significant.

Results: No statistical differences in age and BMI were found between the three groups.

No statistical differences in any sleep parameter were found between cervical and dorsal SCI patients. Tetraplegics and paraplegics compared to controls presented: a significantly lower proportion in N1, N3 and N4 NREMS, and significantly higher percentage of N2 NREMS.

Paraplegics, presented a significantly lower proportion of REM sleep compared to controls.

Tetraplegics compared to controls, but not to paraplegics, presented a higher number of arousal ad arousal index. Due to the elevated intra-subject variability no statistical differences were observed in PLMS and PLMS index between the three groups.

Conclusion: The results of the present study indicate compromised sleep in patients with cervical and thoracic SCI with an augmented proportion N1 NREMS and reduced deep NREMS compared to controls. Cervical SCI, compared to controls, had an increased number of arousal and Arousal Index, which were not related to PLMS.

References:

1. Adey WR, et al. Arch Neurol. 1968;19:377.

2. Jensen MP, et al. Rehabil Psychol. 2009;54:323.

3. Scheer FA, et al. Spinal Cord. 2006;44:78.

\section{Free Communications}

Thursday, 13th October, 2011, 09:15-10:00 h

\section{FC5}

\section{Abnormal gastric myoelectrical activity in postural tachycardia syndrome (PoTS)}

Seligman $H^{l}$, Low $A^{l}$, Asahina ${ }^{4}$, Mathias $J^{l}$

${ }^{1}$ Autonomic and Neurovascular Medicine Unit, Imperial College, London, United Kingdom. ${ }^{2}$ Department of Physiology, Anatomy and Genetics, University of Oxford, Oxford, United Kingdom.

${ }^{3}$ Autonomic Unit, National Hospital of Neurology and Neurosurgery, London, UK. ${ }^{4}$ Department of Neurology, Chiba University School of Medicine, Chiba, Japan

Postural Tachycardia Syndrome (PoTS) is a syndrome in which patients present with inappropriate orthostatic tachycardia and orthostatic intolerance. In addition to postural symptoms, many PoTS patients have allied features, including gastrointestinal (GI) symptoms, which can be severely debilitating. While the pathophysiology of orthostatic symptoms in PoTS is increasingly well understood, 
little is known of the pathophysiology underpinning the GI symptoms.

Aim: The aim of this study was, therefore, to evaluate gastric myoelectrical activity before and after food ingestion in PoTS.

Methods: Using surface electrogastrography, we examined the myoelectrical activity [dominant frequency and its instability coefficient (ICDF)] of gastric slow waves before and after standard liquid meal ingestion in 15 PoTS patients ( 1 male and 14 females, $27 \pm 1$ years) with and without GI symptoms and in 11 healthy individuals ( 3 males and 8 females, $23 \pm 2$ years).

Results: Compared to healthy individuals, all PoTS patients had irregular gastric myoelectrical activity both before and after meal ingestion. ICDF was elevated in PoTS before $(8.8 \pm 1.5$ vs. $4.0 \pm 1.0 \%$ in the healthy group, $\mathrm{P}<0.05)$ and $30-45 \mathrm{~min}$ after $(10.0 \pm 2.0$ vs. $4.0 \pm 1.1 \%, \mathrm{P}<0.05)$ the meal. Patients presenting with GI symptoms had more severe gastric dysrhythmia than patients without GI symptoms. ICDF returned to pre-meal levels 30-45 min after the meal in the patients without GI symptoms $(5.6 \pm 1.5 \%)$ but it remained markedly elevated in those with GI symptoms $(15.0 \pm 2.0 \%, \mathrm{P}<0.05$ vs. healthy and PoTS without GI groups).

Conclusions: These findings suggest that PoTS patients have irregular gastric myoelectrical activity similar to that seen in patients with functional GI disorders, e.g., functional dyspepsia, and that PoTS patients with GI symptoms have more severe gastric dysrhythmia than those without GI symptoms. Overall, this work suggests that there might be a more widespread abnormality of autonomic control mechanisms in PoTS than previously thought.

\section{FC6}

\section{Effects of transcranial direct current stimulation} (TDCS) on esophageal motility and pathological waves in gastroesophageal reflux disease (GERD) patients

\author{
Vigneri $S^{l}$, Bonventre $S^{l}$, Inviati $A^{l}$, Cosentino $G^{l}$, Giglia $G^{l}$, \\ Puma $A^{l}$, Brighina $F^{l}$, Fierro $B^{l}$ \\ ${ }^{1}$ University of Palermo, Palermo, Italy
}

Aims: To evaluate, through esophageal manometry, the effects of transcranial direct current stimulation (TDCS) on esophageal mean distal waves amplitude, pathological distal waves and lower esophageal sphincter (LES) mean pressure in patients with GERD.

Methods: We studied 16 patients with clinical diagnosis of GERD who previously underwent endoscopy to differentiate erosive reflux disease (ERD) from non-erosive reflux disease (NERD). We performed an esophageal manometry before and during cortical stimulation with TDCS $(2 \mathrm{~mA})$ on the right esophageal motor area [1]. Randomly nine patients were assigned to anodal stimulation, seven patients to sham stimulation. We asked each patient to swallow a $5 \mathrm{ml}$ water bolus for ten subsequent times, distal waves amplitude (considered as distal amplitude $<30 \mathrm{mmHg}$ or as not propagated distal peristalsis), number of pathological waves (over 10 swallows) were measured 5 and $3 \mathrm{~cm}$ over the esophageal sphincter. LES pressure was obtained as well. In order to compare mean distal waves amplitude, number of distal pathological waves and mean basal LES pressure, before and during TDCS in both groups of patients, statistical analysis was performed by repeated measures ANOVA with Group (two levels: anodal-sham) as between subject factor, Time (two levels: before-during TDCS) and Swallows (ten levels) as within subject factors.

Results: Mean distal waves amplitude increased significantly $(\mathrm{p}=0.01)$ and the number of distal pathological waves decreased significantly $(\mathrm{p}=0.03)$ during anodal TDCS, while sham stimulation did not influence both parameters. LES mean pressure was significantly decreased during anodal $(\mathrm{p}=0.05)$ but not during sham stimulation in our patients.

Conclusions: although preliminary, our data suggest that TDCS can influence cortical control of esophageal motility and reduce pathological motor pattern in GERD patients.

\section{Reference:}

1. Aziz Q, et al. Gastroenterology. 1996;111:855-62.

\section{FC7}

\section{Segmental weighing reveals normal and abnormal splanchnic vasoconstriction upon cardiac preload lowering}

Wolf $\mathrm{JP}^{l}$, Bouhaddi $M^{1}$, Berger $E^{3}$, Magy-Bertrand $N^{4}$, Thiriez $G^{5}$, Mourot $L^{2}$, Degano $B^{l}$, Regnard $J^{l}$

${ }^{1}$ University Hospitals, Physiology Department, Besançon, France.

${ }^{2}$ Université de Franche Comté, EA3920, Besançon, France.

${ }^{3}$ University Hospitals, Neurology Department, Besançon, France.

${ }^{4}$ University Hospitals, Internal Medicine Department, Besançon,

France. ${ }^{5}$ University Hospitals, Pediatric Department, Besançon,

France

Aims: Physiological adjustments of vascular capacity in the legs and the splanchnic region are pivotal to efficient hemodynamic control, e.g. to changes from supine to upright. Cardiac preload lowering in POTS has been ascribed to abnormal vasoconstrictive behaviour either in legs or splanchnic vasculature [1]. Easy recognition of the vascular bed with impaired vasomotor control would help chossing appropriate therapeutic means.

Methods: $\mathrm{A} \ll$ segmental weighing $\gg(\mathrm{SW})$ device was designed to track continuous changes in leaning forces of 1-legs, 2-abdomen/ pelvis region and 3-thorax of supine subject. During SW graded thigh venous compression (TVC) is used to retain blood in the legs. Time-scaled changes in leg leaning force reflect blood pooling and quantify both leg venous compliance and arterial inflow with patterns similar to strain gauge calf plethysmography [2]. Pharmacological changes in venous and arterial tone were assessed in both healthy subjects and patients with Parkinson's disease [2]. With TVC, the lower trunk leaning force (abdomen/pelvis, A) lessens during the increase of leg blood content (L). With graded TVC, comparison of A alleviations with L loadings shape a relationship which reflects activation of splanchnic vasoconstriction.

Results: In nine healthy subjects, the ratio of A force lessening to increasing $\mathrm{L}$ force was similar on 3 different days with normal baseline hydration (values around 1.1-1.2), but was significantly increased $(>1.3, \mathrm{p}<0.05)$ when hypovolemia had been achieved and led to decreased leg venous distensibility $(\mathrm{p}<0.01)$. Conversely, the ratio was largely lowered (below 0.8 ) when the splanchnic vasoconstriction was impeded (cirrhosis with hepato-renal syndrome). We used SW and TVC procedures in persons in whom tilt testing revealed POTS and neurogenic orthostatic hypotension. In various patterns of orthostatic intolerance, decreases in cardiac preload were found associated with impaired splanchnic vasoconstriction/or enlarged leg venous compliance/or lowered leg arterial inflow.

Conclusions: Segmental weighing and TVC data easily provide non invasive delineation of vasomotor abnormalities and support chossing therapeutic means in various conditions of orthostatic intolerance.

References:

1. Stewart JL. Am J Physiol Heart Circ Physiol. 2004;287:H319.

2. Wolf JP, et al. Clin Sci. 2006;110:369. 


\section{Free Communications}

\section{Thursday, 13th October, 2011, 11:15-12:00 h}

\section{FC8}

Evaluation of the autonomic nervous system in patients with stage IV incurable, advanced solid tumours

\author{
Hundsberger $T^{2}$, Vehoff $\mathrm{J}^{l}$, Haegele-Link $S^{l}$, Tettenborn $B^{l}$, \\ Strasser $F^{l}$ \\ ${ }^{1}$ Cantonal hospital, Department of neurology, St. Gallen, Switzerland. \\ ${ }^{2}$ Cantonal Hospital, Division of Hematology and Oncology, \\ St., Gallen, Switzerland
}

Introduction: Anorexia-cachexia syndrome (ACS) is found in up to $80 \%$ of patients suffering from stage IV solid tumours. It is characterized by weight loss, reduced food intake and systemic inflammation. Primary ACS seems to be a complex paraneoplastic syndrome evolving throughout the course of disease progression and treatment. Studies investigating the impairment of the autonomic nervous system as a contributing factor of ACS in these patients are rare.

Methods: Patients suffering from incurable stage IV solid tumors were evaluated clinically, with an autonomic test battery and nerve conduction studies in two prospectively trials (GUBRA-CAT, Grehlin). Autonomic testing consisted of a time domain based analysis of heart rate variability under the paradigms of breathing (at rest and deep breath) for the evaluation of the parasympathetic cholinergic (PC) part of the autonomic system, blood pressure changes following and valsalva manoeuvre (VM) (qualitative and quantitative evaluation of the sympathetic noradrenergic ( $\mathrm{SN}$ ) system using the reactive overshoot of blood pressure in phase IV after VM) and active standing (orthostasis) with the Finometer Pro (FP) device (Finapres Medical Systems, The Netherlands). In addition the sympathetic skin response (SSR) was done for the evaluation of the sympathetic cholinergic (SC) system.

Results: 13 patients suffering from NSCLC $(n=5)$, GI-tumours $(\mathrm{n}=3)$ and various tumours $(\mathrm{n}=6)$ were evaluated. 11 of 13 patients showed pathological results in two categories of which SN $(n=6)$, SC $(n=6)$ and orthostatic hypotension $(n=5)$ were equally affected. Of note, only one patient showed pathological results in PC category. 8 of 13 patients additionally showed subclinical large fibre polyneuropathy only two of them being previously treated with neurotoxic chemotherapy.

Conclusion: In our small cohort of patients with advanced solid tumours autonomic dysfunction occurs frequently in the sympathetic nervous system and includes orthostatic hypotension but does rarely affect the parasympathetic cholinergic part of the autonomic nervous system.

\section{FC9 \\ Hemisphere-specific dysfunction of central cardiovascular control in ischemic stroke patients}

\author{
Moeller $S M^{1}$, Koehn $J K^{1}$, Akhundova $A A^{1}$, De Fina $P D F^{2}$, \\ Marthol HM ${ }^{1}$, Schwab SS ${ }^{1}$, Hilz MJH \\ ${ }^{1}$ Department of Neurology, University of Erlangen-Nuremberg, \\ Erlangen, Germany. ${ }^{2}$ International Brain Research Foundation, \\ Edison, USA. ${ }^{3}$ Departments of Neurology, Medicine, and Psychiatry, \\ New York University, New York, USA
}

Background: We have recently shown that autonomic dysfunction correlates with clinical severity of stroke (Hilz et al. 2011). So far, reports regarding autonomic dysfunction differences after left- or right-sided stroke are inconsistent.

Objective: To assess whether there are hemisphere-specific correlations between autonomic dysfunction and stroke severity.
Methods: In 29 left middle cerebral artery (MCA)-stroke patients $(67 \pm 13$ years) and 27 right MCA-stroke patients ( $69 \pm 15$ years) we assessed National Institutes of Health Stroce Scale (NIHSS) scores and autonomic cardiovascular parameters within $24 \mathrm{~h}$ after stroke onset. From 5-min RR-intervals (RRI) and blood pressure (BP) recordings, we calculated spectral powers of mainly sympathetically mediated low- (LF $0.04-0.15 \mathrm{~Hz}$ ) and parasympathetically mediated high-frequency- (HF $0.15-0.5 \mathrm{~Hz}$ ) RRI oscillations, RRI-LF/HFratios indicating sympatho-vagal balance, sympathetically mediated LF-powers of BP-oscillations, and baroreflex sensitivity (BRS) as gain between RRI- and systolic BP-oscillations for coherence $>0.7$. We used $t$ tests to assess differences between left- and right-MCA stroke patients and the Spearman rank correlation test to correlate autonomic cardiovascular parameters with NIHSS scores. Significance was assumed for $\mathrm{p}<0.05$.

Results: Left MCA-stroke patients had lower RRIs and RRI-HFpowers but higher BPdia and RRI-LF/HF-ratios than right MCAstroke patients. In left MCA-stroke patients NIHSS-scores directly correlated with RRI-LF/HF-ratios but inversely correlated with RRIs, RRI-HF-powers, and BRS. In right MCA-stroke patients NIHSSscores inversely correlated with RRI-HF-powers. Spearman-Rhovalues ranged from 0.38 to 0.49 .

Conclusions: Correlations between stroke severity and autonomic dysfunction are hemisphere-specific. Particularly left MCA-stroke patients had decreasing parasympathetic tone and BRS, and a shift towards sympathetic dominance with increasing stroke severity. Stroke induced hypoactivity of lesioned central autonomic network structures might account for the observed hemisphere-specific differences of correlations between stroke severity and autonomic dysfunction.

Acknowledgment: The study was supported by the Rolf- and Hubertine-Schiffbauer-Foundation, Hof, Germany, and the International Brain Research Foundation Inc., Edison, NJ, USA.

Reference:

1. Hilz, et al. Stroke. 2011:6:1528-33.

\section{FC10}

\section{Local head-cooling increases cardiovagal and peripheral sympathetic activity}

\author{
Koehn $J K^{1}$, Cimpianu $C L C^{1}$, Kallmuenzer BK ${ }^{1}$, Kollmar $R K^{1}$, \\ Pauli $\mathrm{EP}^{l}$, Moeller $\mathrm{SM}^{1}$, Hilz $\mathrm{MJH}^{2}$ \\ ${ }^{1}$ Department of Neurology, University of Erlangen-Nuremberg, \\ Erlangen, Germany. ${ }^{2}$ Departments of Neurology, Medicine, \\ Psychiatry, New York University, New York, NY, USA
}

Introduction: Therapeutic hypothermia is an effective treatment of cerebral ischemia and edema, e.g. due to trauma or stroke. Yet, effects of head-cooling on cardiovascular autonomic function have not been evaluated.

Aim: To determine cardiovascular autonomic changes during local head-cooling.

Methods: In ten healthy men ( $35 \pm 13$ years), we applied headcooling using a head- and neck-cooling device (HVM medical, Germany). Rectal, tympanic and skin temperature was measured continuously. In each participant, we assessed 5-min mean values and standard deviation of RR-intervals (RRI), systolic, diastolic blood pressures (SBP, DBP) and laser Doppler skin blood flow [SBF; in perfusion units $(\mathrm{PU})]$ at the right index finger pulp, before and after $60 \mathrm{~min}$ of head-cooling. We calculated spectral powers of mainly sympathetic low- (LF $0.04-0.15 \mathrm{~Hz}$ ) and parasympathetic high-frequency (HF $0.15-0.5 \mathrm{~Hz}$ ) RRI-oscillations and sympathetic LFpowers of BP-oscillations. We compared values before and during head-cooling (ANOVA, post-hoc analysis; significance: $\mathrm{p}<0.05$ ).

Results: Head-cooling significantly increased DBP (64.8 \pm 5.0 vs. $72.9 \pm 6.0 \mathrm{mmHg})$, RRIs $(983.4 \pm 192.0$ vs. $1,101.5 \pm 231.2 \mathrm{~ms})$ 
and RRI-HF-powers $\left(464.9 \pm 289.1\right.$ vs. $\left.1.271 .6 \pm 1.072 .0 \mathrm{~ms}^{2}\right)$, and decreased SBF $(189.7 \pm 84.9$ vs. $33.8 \pm 20.8 \mathrm{PU})$, rectal, tympanic and skin temperature. SBP increased insignificantly $(116.8 \pm 7.0 \mathrm{vs.}$ $125.9 \pm 9.1 \mathrm{mmHg}$ ), RRI-LF-powers and LF-powers of BP remained unchanged during head-cooling.

Conclusion: The decrease in SBF and increase in DBP during headcooling indicates sympathetically mediated peripheral vasoconstriction. Increased RRIs and RRI-HF-powers reveal cardiovagal activation. Thus, head-cooling has effects similar to the cold face test (Hilz et al. 1999). Cardiovagal activation may benefit stroke or brain injury patients by counterbalancing their increased sympathetic activity.

Reference:

1. Hilz, et al. Am J Physiol. 1999:276:R1833-9.

\section{Free Communications \\ Thursday, 13th October, 2011, 14:45-15:30 h}

\section{FC11}

\section{Are 10 min of orthostatic postural challenge useful to detect an orthostatic hypotension in MSA patients?}

\author{
Pavy-Le Traon $A^{1,2}$, Meissner $W^{l}$, Piedvache $A^{l}$, Tison $F^{l}$, Rascol $O^{l}$ \\ ${ }^{1}$ French reference center of MSA, Toulouse University Hospital \\ and Bordeaux University Hospital, France. ${ }^{2}$ INSERM, U-1048, \\ Toulouse, France
}

Multiple system atrophy (MSA) is a rare neurodegenerative disease characterised by the association of Parkinsonian or cerebellar syndrome and dysautonomia (MSA P or C). To detect orthostatic hypotension $(\mathrm{OH})$ is particularly important in MSA since $\mathrm{OH}$, as a feature of autonomic failure, is one of the main diagnosis criteria [1]. However, orthostatic symptoms have low sensitivity and to detect $\mathrm{OH}$ may be difficult. In a recent paper, Jammadas-Khoda [2] showed that, in Parkinson disease (PD), $\mathrm{OH}$ could be delayed and often occurred after the $3 \mathrm{~min}$ in upright position usually recommended for $\mathrm{OH}$ detection.

The main objective of this work was to evaluate the interest of a 10 min orthostatic test to detect $\mathrm{OH}$ in MSA patients. We analysed 20010 min-stand tests in 141 MSA patients of the French Reference Center (85 MSA-P and 56 MSA-C/24 possible-117 probable). These patients had at least one orthostatic test with every minute measurement of arterial blood pressure (ABP) and heart rate (HR). We calculated the number of patients who had $\mathrm{OH}$ (defined as fall of Systolic BP $\geq 30 \mathrm{mmHg}$ and/or diastolic BP $\geq 15 \mathrm{mmHg}$ (according to the revised MSA criteria)) within 3, 5 and 10 min of standing.

$\mathrm{OH}$ occurred in 146/200 tests. In 54 tests, no significant $\mathrm{OH}$ occurred. Among these 146 positive tests, $\mathrm{OH}$ became significant between min 3 and $\min 10$ in 40 patients (27\%). OH was significantly correlated with disease duration and age but not with disease type (C or P). These data showed the interest of a $10 \mathrm{~min}$ stand test to detect $\mathrm{OH}$ in MSA patients. This work is currently continued within the frame of the EMSA Group. References:

1. Gilman, et al. Neurology. 2008:71(9):670-6.

2. Jammadas-Khoda. Mov Disord. 2009:24(12):1747-51.

\section{FC12}

\section{3,4-diaminopyridine improves orthostatic hypotension}

Singer $W^{l}$, Opfer-Gehrking $T L^{1}$, Gehrking $J A^{l}$, Figueroa $J J^{I}$, Sandroni $P^{I}$, Low $P A^{I}$

${ }^{1}$ Mayo Clinic, Rochester, USA
Background/aims: Treatment options for neurogenic orthostatic hypotension $(\mathrm{OH})$ are limited and often associated with aggravation of supine hypertension. We could recently demonstrate that acetylcholinesterase inhibition is efficacious in $\mathrm{OH}$ without worsening of supine hypertension, but with modest efficacy. We now hypothesized that the potassium channel blocker 3,4-diaminopyridine (3,4-DAP) by enhancing neurotransmitter release-has similar but greater beneficial effects in patients with $\mathrm{OH}$, as it should not only enhance adrenergic ganglionic neurotransmission but also neurotransmission at the adrenergic endorgan synapse, resulting in serially linked and therefore potentiated effects under baroreflex control. Our aims with this pilot study were to prove the hypothesis that 3,4-DAP effectively improves orthostatic blood pressure (BP) and symptoms in $\mathrm{OH}$ with predominant effects in the upright position.

Methods: We studied nine patients with neurogenic $\mathrm{OH}$ in a placebocontrolled patient-blinded protocol. Following a $20 \mathrm{~min}$ period of supine rest, BP and heart rate (HR) were continuously monitored for a $10 \mathrm{~min}$ period in the supine position, followed by $5 \mathrm{~min}$ of 70 degree head-up tilt, before and $1 \mathrm{~h}$ after oral administration of $20 \mathrm{mg}$ of 3,4DAP. Supine and orthostatic averages of parameters were obtained and along with orthostatic symptoms compared between treatment status. QTc interval and side effects were recorded for $2 \mathrm{~h}$ following medication administration.

Results: Orthostatic BP was significantly improved after administration of 3,4-DAP compared to placebo (systolic BP $112.1 \pm 6.7$ vs. $91.8 \pm 6.6 \mathrm{mmHg}, \mathrm{p}=0.001$ ). Similar, highly significant changes were also seen for diastolic BP. Orthostatic symptoms improved significantly $(2.7 \pm 1.0$ vs. $5.2 \pm 1.1)$ on a 10 point visual-analog scale. Supine BP also increased significantly (systolic BP $167.3 \pm 6.6$ vs. $151.4 \pm 5.8 \mathrm{mmHg}$ ), but less than orthostatic BP. Only one patient reported modest side effects. There were no significant effects on HR. QTc did not increase significantly.

Conclusions: 3,4-DAP is safe and well tolerated, and dramatically improves $\mathrm{OH}$ and orthostatic symptoms. The improvement is much more pronounced than that seen with pyridostigmine, consistent with the hypothesis of potentiated, serially linked effects. A double-blind, placebo-controlled trial is now planned to confirm these findings.

\section{FC13}

\section{Frequent syncope is a risk factor for white matter lesions in migraineurs and controls}

Kruit $M C^{l}$, Thijs $R D^{l}$, Ferrari $M D^{l}$, Launer $L J^{2}$, van Buchem $M A^{l}$, van Dijk $J G^{l}$

${ }^{1}$ Leiden University Medical Centre, Leiden, Netherlands.

${ }^{2}$ National Institutes of Health, Bethesda, USA

Objective: We and others showed that migraineurs are at increased risk of white matter lesions (WML), subclinical cerebellar infarct-like lesions, and clinical stroke. Migraineurs also have a higher prevalence of frequent syncope and orthostatic intolerance (OI). This study assesses whether frequent syncope and OI may increase the risk of WML independent from migraine.

Methods: Migraineurs $(\mathrm{n}=291)$ and controls $(\mathrm{n}=140)$ from the population-based CAMERA cohort (aged 30-60 years, and free of other neurological symptoms) underwent: (1) brain MRI scan, (2) structured telephone interview including questions on frequent syncope ( $>5 /$ lifetime $)$ and OI, and (3) physical examination with blood pressure and heart rate measurements, assessing orthostatic hypotension $(\mathrm{OH})$ and postural tachycardia syndrome (POTS). 
Results: Frequent syncope $(\mathrm{OR}=2.7,95 \% \mathrm{CI} 1.3-5.5)$ and $\mathrm{OI}$ $(\mathrm{OR}=2.0$ [1.1-3.6]) were independent risk factors for high load of deep white matter lesions (DWML). Effects were strongest in women and similar in migraineurs and controls. Migraine diagnosis did not mediate or moderate these associations. Female migraineurs without frequent syncope or OI still were at risk of high DWML-load (OR = 2.3 [1.0-5.3]). Individuals with OI had higher prevalence of high periventricular WML-load (2 vs. $8 \%$, $\mathrm{p}=0.005$ ). Individuals with $\mathrm{OH}$ had higher prevalence of infratentorial hyperintense lesions ( 2 vs. 9\%, p = 0.005). Syncope and OI were not related to subclinical infarcts or infratentorial lesions.

Interpretation: Frequent syncope, OI and migraine independently increase the risk of WML, in particular in females. OH increased the risk of infratentorial hyperintense lesions.

\section{Free Communications \\ Thursday, 13th October, 2011, 16:45-17:30 h}

\section{FC14}

\section{Peripheral chemoreflex hyperactivity inhibits arterial baroreflex in heart failure}

Despas $F^{l}$, Lambert $E^{3}$, Vaccaro $A^{l}$, Labrunee $M^{1}$, Franchitto $N^{l}$, Castel $M^{l}$, Lebrin $M^{1}$, Galinier $M^{4}$, Senard $J M^{l}$, Pathak $A^{l}$

${ }^{1}$ Institut National de la Sante et de la Recherche Médicale (INSERM) U1048, Département de Médecine Cardiovasculaire, Institut de Médecine Moléculaire de Rangueil, IFR31, Université Paul Sabatier, Toulouse, France. ${ }^{2}$ Département de Pharmacologie Clinique, Centre Hospitalier Universitaire, Toulouse, France. ${ }^{3}$ Human

Neurotransmitter Laboratory, Baker IDI Heart and Diabetes Institute, Melbourne, Australia. ${ }^{4}$ Département de Cardiologie, Centre Hospitalier Universitaire, Toulouse, France

Aim: Heart failure (HF) is characterized by a decreased activity of sympathetic baroreflex, whose mechanisms are partially understood. Peripheral chemoreceptors may contribute to the decreased activity of the sympathetic baroreflex in patients with HF. The aim of the study was to investigate the role of chemoreflex hyperactivity on sympathetic baroreflex in HF.

Methods: We compared the gain of sympathetic baroreflex in two groups of HF patients: with and without activation of their peripheral chemoreceptors. Secondarily, in a double-blind randomized trial versus ambient air, we examined the effect of a chemoreflex inactivation by pure oxygen $(100 \%$ for $15 \mathrm{~min})$ on the baroreflex sympathetic gain [relationship between sympathetic nerve activity (MSNA) and diastolic blood pressure] in HF patients with high $(\mathrm{n}=18)$ or normal $(\mathrm{n}=20)$ chemosensitivity.

Results: Basal MSNA values were higher $(60.6 \pm 3.2$ vs. $48.9 \pm$ 3.7 bursts/min, $\mathrm{p}<0.05$ ) and sympathetic baroreflex gain decreased $(3.06 \pm 0.55$ vs. $5.51 \pm 0.69$ bursts $\% / \mathrm{mmHg}, \quad \mathrm{p}<0.05)$ in $\mathrm{HF}$ patients with increased peripheral chemosensitivity compared with HF patients with normal chemosensitivity. Administration of $100 \%$ oxygen leads to a significant decrease in MSNA (from $60.5 \pm 3.2$ to $52.6 \pm 3.2$ bursts/min, $\mathrm{p}<0.001$ ) and significantly increased the gain of the baroreflex (from $2.95 \pm 0.56$ to $6.18 \% \pm 0.77$ bursts/ $\mathrm{mmHg}, \mathrm{p}<0.001)$ in HF patients with increased chemosensitivity. On the other side, in HF patients with normal chemosensitivity neither ambient air nor $100 \%$ oxygen changed MSNA, hemodynamic parameters nor sympathetic baroreflex gain.
Conclusions: We identified a new mechanism responsible for the decreased baroreflex gain in HF: increased activity of peripheral chemoreflex. Peripheral chemoreflex hyperactivity contributes to sympathetic activation and reduced sympathetic baroreflex gain in HF. This interaction may explain how peripheral chemoreceptors can contribute to the poor prognosis of HF patients.

\section{FC15}

Tako-Tsubo cardiomyopathy: direct evidences of sympothetic nervous system hyperactivity and baroreflex impairment

Despas $F^{l}$, Vaccaro $A^{l}$, Delmas $C^{2}$, Lebrin $M^{1}$, Lairez $O^{2}$, Galinier $M^{2}$, Senard $\mathrm{JM}^{1}$, Pathak $A^{1}$

${ }^{1}$ Institut National de la Santé et de la Recherche Médicale (INSERM), U1048, Département de Médecine Cardiovasculaire, Institut de Médecine Moléculaire de Rangueil, IFR31, Université Paul Sabatier, Toulouse, France. ${ }^{2}$ Département de Cardiologie, Centre Hospitalier Universitaire de Toulouse, Toulouse, France

Aims: Tako-Tsubo cardiomyopathy (TTC) is an acute reversible condition that involves left ventricular apical 'ballooning' and mimics acute myocardial infarction with no detectable coronary arterial disease. TTC typically affects aged postmenopausal women and is usually triggered by profound emotional or physical stress. The exact physiopathology remains unknown but datas suggest a link between sympathetic hyperactivity (catecholamine plasmatic level, heart rate variability) and TTC. Autonomic control of the cardiovascular system may be altered in TTC and a possible link between baroreflex dysfunction and TTC has been proposed. Up to now sympathetic hyperactivity has not been established with a technique directly measuring the sympathetic nervous system (SNS) and baroreflex function has never been assessed in TTC patients. The aim of our study was to determine post-ganglionic muscle sympathetic nerve activity (MSNA) by microneurography and spontaneous arterial baroreflex function in TTC patients compared to matched heart failure control patients.

Methods: We enrolled 13 TTC patients $(80.1 \pm 2.1$ years, all female, left ventricular ejection fraction $40 \pm 2 \%$ ) and 13 control patients matched for age, sex, LVEF, renal function and hemoglobinemia. $36 \mathrm{~h}$ after admission, all patients underwent a microneurography with assessment of the arterial baroreflex gain (slope of the regression line that represents the relationship between MSNA and diastolic blood pressure).

Results: There were no differences between groups on hemodynamics parameters (SBP, DBP, MBP, HR and oxygen saturation). TTC patients presented a sympathetic activity significantly increased (MSNA $66.3 \pm 2.7$ vs. $55.6 \pm 2.6$ bursts $/ \mathrm{min} ; \mathrm{p}=0.0088$ ) and arterial baroreflex gain significantly decreased $(1.2 \pm 0.3$ vs. $2.5 \pm 0.4 \%$ bursts/ $\mathrm{mmHg} ; \mathrm{p}=0.005$ ) in comparison with control patients.

Conclusions: This study showed for the first time with a technique, which directly assesses SNS activity, that TTC patients present a sympathetic hyperactivity and an impairment of arterial baroreflex.

\section{FC16}

\section{The impact of gender and training volume on autonomic modulations in non-elite endurance athletes}

Wilhelm $M^{1}$, Fürholz $M^{l}$, Roten $L^{1}$, Tanner $H^{l}$, Schmid JP $P^{l}$, Saner $H^{1}$ ${ }^{1}$ University of Bern, Department of Cardiology, Bern, Switzerland 
Background: The risk of sudden cardiac death is increased in athletes with a striking male predominance. Sympathetic activation precipitates or enhances ventricular arrhythmias, whereas vagal tone suppresses their occurrence. We studied the impact of gender and training volume on autonomic modulation in non-elite endurance athletes.

Methods: Amateur athletes scheduled to participate in the 2010 Grand Prix of Bern, a 10 Mile race, were invited. 873 runners applied for participation, of whom 70 female and 70 male athletes were randomly selected. Runners with cardiovascular diseases were excluded. Athletes were stratified according to their average weekly training hours of the last 3 months in a low volume $(\leq 4 \mathrm{~h})$ and a high volume $(>4 \mathrm{~h})$ training group. Echocardiography and $24 \mathrm{~h}$ Holter monitoring with frequency domain analysis of heart rate variability was performed. Sex and training volume groups were compared.

Results: 114 athletes were included in the final analysis. There were no gender differences (female vs. male) for age ( $43 \pm 7$ vs. $41 \pm 7$ years; $\mathrm{P}=0.102), 10$ Mile race time $(82 \pm 11$ vs. $79 \pm 11$ min.; $\mathrm{P}=0.116)$, average weekly training hours $(5.8 \pm 2.6$ vs. $5.2 \pm 3.1 \mathrm{~h} ; \mathrm{P}=0.289)$, former marathon participations $(5.7 \pm 6.5$ vs. $4.9 \pm 7.4 ; \mathrm{P}=0.124)$, and premature ventricular beats $(9 \pm 23$ vs. $50 \pm 290 ; \mathrm{P}=0.359)$. Left ventricular systolic and diastolic function were normal in all athletes. The sympatho-vagal balance, represented as the low frequency (LF)/high frequency (HF) power ratio, was significantly lower in female athletes during daytime and nighttime (Fig. 1). In female athletes, a higher training volume was associated with a significantly lower LF/HF power ratio at nighttime, while in male athletes, the same was true at daytime (Fig. 1).

Conclusions: For a comparable amount of training and performance, female athletes showed a higher vagal tone, possibly protecting against ventricular arrhythmias. Female and male athletes showed a different circadian pattern of the training-related increase in vagal tone.

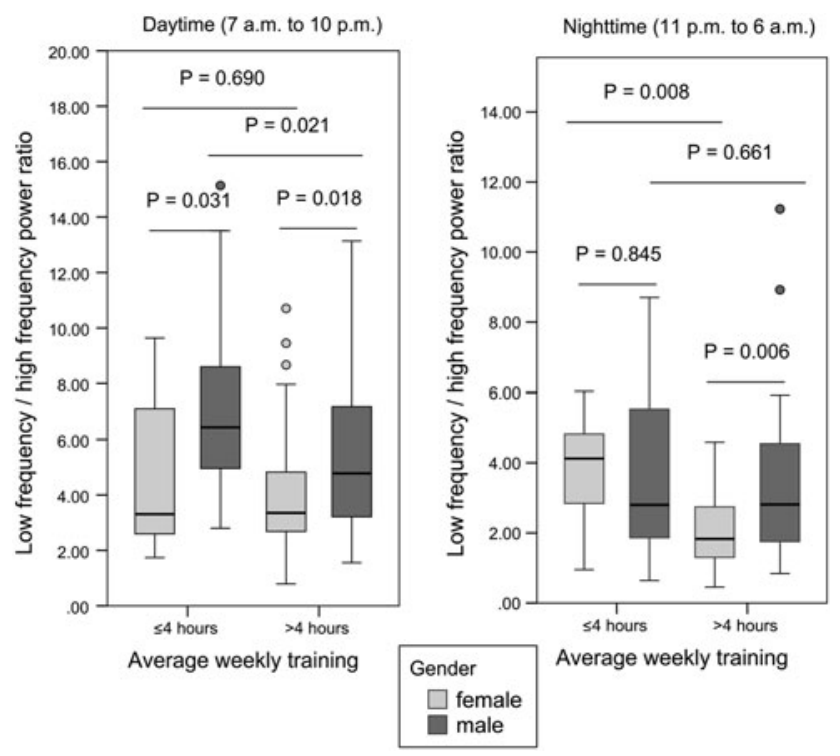

Fig. 1 Gender and training volume differences of sympatho-vagal balance

\section{Posters}

\section{P01}

Assessment of baroreflex sensitivity and time domain HRV parameters before and after carotid baroreflex activation in three patients with resistant hypertension

Testa $E^{l}$, Berra $E^{l}$, Covella $M^{l}$, Totaro $S^{l}$, Fulcheri $C^{l}$, Fabbri $A^{l}$, Maule $S^{1}$, Di Stefano $C^{l}$, Rabbia $F^{l}$, Veglio $F^{l}$, Testa $E^{2}$

${ }^{1}$ Department of Medicine and Experimental Oncology, Hypertension Unit, University of Turin, Turin, Italy. ${ }^{2}$ SCDU Medicina 4, AOU S. Giovanni Battista, Corso Bramante 88, 10126, Torino, Italy;

Phone: +39-0116336959; Fax: +39-0116336931;

e-mail: elisatesta82@gmail.com

Aim: Recent technical advances have renewed interest in devicebased therapy for drug-resistant Hypertension therapy. Findings from recent clinical studies regarding the efficacy of electric stimulation of the carotid sinus (BAT) are consistent with a good response rate. However, tests able to predict the clinical results of the implant are not available at present.

Methods: We assessed spontaneous baroreflex sensitivity (BRS) by using non-invasive beat to beat device Portapres, time domain HRV parameters by using $24 \mathrm{~h}$ ecg holter and BP parameters (both office and $\mathrm{ABPM}$ ) in three patients (3F, age range $40-53$ years) with resistant hypertension in poli-pharmacotherapy (mean number of antihypertensive drugs $7 \pm 2$ ) before and after 1, 3, 6 months after electrical activation of the carotid baroreceptors (Rheos HT, CVRx, Minneapolis, MN, USA).

Results: Mean office BP values before BAT were 221/130 \pm 10 / $13 \mathrm{mmHg}$, and were not significantly reduced after 3 months (220/ $115 \pm 10 / 6), 6$ months $(220 / 115 \pm 17 / 15)$. BRS was $5.78 \pm 1.1$ at baseline and was unchanged after $3(5.1 \pm 1.1)$ and 6 months $(5.2 \pm 1.2)$, similar results were found for HRV parameters such as SDNN (79 \pm 15 at baseline, $55 \pm 17$ at 6 months), SDANN ( $55 \pm 21$ at baseline, $48 \pm 21$ at 6 months), by contrast NN50 and RMSSD, parameter related to vagal tone, were increased in two of the three patients. BRS increased greater immediately after the beginning of the electric stimulation, decreasing in the following hours/days. In one patient, for example, after four hours of electric stimulation the BRS was $9.1 \mathrm{~ms} / \mathrm{mmHg}$, and decreased to $4.1 \mathrm{~ms} / \mathrm{mmHg}$ after 2 days BAT

Conclusion: BRS was impaired at baseline (compared to age and sex related standards) and unchanged after 6 months in these patients unresponsive to BAT therapy. Even if there are not similar evaluations in other studies of BAT therapy it may be hypothesized that an impaired BRS sensitivity at baseline may be associated to a poor response to BAT therapy.

\section{P02}

Autoimmune autonomic ganglionopathy associated with voltage-gated potassium channel antibodies

Singer $W^{l}$, McKeon $A^{l}$, Sletten $D M^{1}$, Low $P A^{1}$

${ }^{1}$ Mayo Clinic, Rochester, MN, USA 
Background: Ganglionic acetylcholine receptor (AChR) autoantibodies have been described in patients with idiopathic autonomic neuropathies. With confirmation and further characterization of the pathophysiologic role of these antibodies, the preferred terminology for this disease category has changed to autoimmune autonomic ganglionopathy (AAG). The majority of patients fulfilling clinical and laboratory criteria for AAG are, however, seronegative for these antibodies and additional pathogenetic antibodies have been postulated. Recently, an association between N-type calcium channel antibodies and AAG has been described in a small number of patients. We now report a case of AAG associated with neuronal voltage-gated potassium channel (VGKC) antibodies.

Methods: A 65 year-old woman with a 1 year history of orthostatic lightheadedness, tachycardia, heat intolerance, diarrhea, and sicca complex underwent comprehensive autonomic evaluation and was diagnosed with AAG. She underwent serial standardized autonomic, clinical, and laboratory evaluations for 2 years, before and during treatment with intravenous immunoglobulin (IVIG).

Results: During her initial evaluation in 2009, the patient was found to have significant anhidrosis (50\% body surface area) and markedly reduced QSART responses with essentially normal cardiovagal and cardiovascular adrenergic function. A paraneoplastic antibody panel was positive for VGKC antibodies at a titer of $0.40 \mathrm{nmol} / \mathrm{l}$, though a search for an occult malignancy remained negative. The patient was treated symptomatically. During subsequent evaluations in 2010, progressive cardiovascular adrenergic impairment was documented with evidence of orthostatic hypotension by December of 2010, associated with a rise in the VGKC antibody titer to $0.77 \mathrm{nmol} / \mathrm{l}$. At that time, treatment with weekly and later biweekly IVIG at $0.4 \mathrm{~g} / \mathrm{kg}$ was initiated. Six months after initiation of IVIG, the patient's autonomic testing was essentially normal with completely normal cardiovascular adrenergic function and minimal remaining hypohidrosis ( $8 \%$ body surface area). This was associated with a decrease in antibody titer to 0.21 and later $0.32 \mathrm{nmol} / 1$.

Conclusions: AAG may be associated with autoimmune antibodies other than the classic ganglionic ACHR and the recently reported calcium-channel antibodies. Whether the VGKC antibodies in our patient have a pathophysiologic role or are nonspecific indicators of an underlying immune process remains subject for further study. The association is, however, important to recognize regardless of the underlying mechanism, considering the remarkable response to immunomodulatory therapy.

\section{P03}

\section{Autonomic evaluation in patients with Fabry disease}

\author{
Aguiar $P^{l}$, Azevedo $O^{2}$, Medeiros $R^{2}$, Araújo $F^{l}$, Ducla Soares $J L^{1}$ \\ ${ }^{1}$ Hospital de Santa Maria, Lisbon, Portugal. ${ }^{2}$ Hospital de Nossa \\ Senhora da Oliveira, Guimarães, Portugal
}

Aims: Evaluation of the autonomous nervous system function in 11 untreated patients with Fabry disease.

Methods: Analysis of the cardiac autonomic reflexes tested by a standard noninvasive method (Ewing protocol) using Task Force Haemodynamic Monitor (CNSystems) technology, and analysis of the sudomotor function performed after pilocarpine stimulation.

Results: Only one patient, the index case, has a mild parasympathetic disfunction (in deep breathing test evaluation). All the others results of the Ewing protocol were within the normal range of our laboratory results. In the sweat test only four patients had normal results and three patients have clearly dimished sudomotor function.
Discussion: This is the largest series of Fabry patients in which standard cardiovascular reflex battery tests was analyzed. No disturbances in cardiovascular autonomic regulation was found in this series, which is in agreement with literature, but this finding cannot be generalized because of heterogeneity of the disease. The slight parasympathetic dysfunction in patient 1 could also be due to chronic renal failure. The abnormal results in the sweat test, like described in the literature, could be due to sympathetic cholinergic disfunction but also because of GB3 deposit in the sweat glands.

\section{P04 \\ Autonomic failure and orthostatic hypotension as early presenting features of Parkinson's disease. A case report}

Milazzo $V^{l}$, Di Stefano $C^{l}$, Servo $S^{l}$, Chiarlo $M^{1}$, Maule $S^{l}$, Veglio $F^{l}$ ${ }^{1}$ Autonomic Unit and Hypertension Unit, Department of Medicine and Experimental Oncology, S. Giovanni Battista Hospital, University of Turin, Turin, Italy. ${ }^{2}$ Department of Neurology, Maggiore della Carità Hospital, Eastern Piedmont University, Novara, Italy

Symptomatic autonomic failure is a common finding in Parkinson's disease (PD). Typically, it appears late during the course of the disease, rarely it can precede the onset of motor symptoms. The degree of orthostatic hypotension $(\mathrm{OH})$ and cardiac sympathetic denervation are not related to the extent of putamen dopaminergic lesions.

We report a case of a 72-year-old male patient, referred to our Unit in 2009. He had been suffering from orthostatic intolerance, syncope and "coat-hanger" back pain during orthostatism or exercise, postprandial hypotension, constipation, nicturia and erectile failure since 2005. ECG, Holter ECG, cardiac catheterization, and echo and CT study of carotid and vertebral arteries were normal.

Autonomic function tests showed a severe autonomic dysfunction with symptomatic $\mathrm{OH}$. Basal plasma adrenaline and noradrenaline were low (49 and $65 \mathrm{pg} / \mathrm{ml}$, respectively) and not responsive to orthostatism (49 and $76 \mathrm{pg} / \mathrm{ml}$, respectively). Electromyography of limbs was normal and other tests excluded a secondary form of autonomic neuropathy. The patient was diagnosed as probable pure autonomic failure (PAF) and therapy with non-pharmacological measures, midodrine and fludrocortisone was started.

Six months later, the patient complained of tremors at rest, affecting the right limbs. The neurologist diagnosed an early extrapyramidal syndrome. The cerebral SPECT-DaT scan showed a reduction of the ligand of dopamine transporter in the left putamen. The cardiac SPECT scan with 123I-metaiodobenzylguanidine showed a cardiac sympathetic denervation (heart-to-mediastinum ratio 1.1 after $4 \mathrm{~h}$ ).

These findings led to the diagnosis of PD with early autonomic involvement, followed by motor symptoms after several years. Therapy with rasegiline was started.

Autonomic failure and $\mathrm{OH}$ can be the presenting clinical features of PD and the clinician should look for signs of parkinsonism during the follow-up of these patients. This report confirms the one-disease hypothesis which considers PAF as a limited form of PD.

References:

1. Goldstein DS, et al. J Neurol Sci. 2011. doi:10.1016/j.jns.2011.04.011.

2. Kaufmann H, et al. Neurology. 2004:63:1093-5. 


\section{P05}

Autonomic nervous system evaluation in patients with systemic lupus erythematosus, comparing active and latent phases of the disease

\author{
Peixoto $L^{l}$, Aguiar $P^{l}$, Espirito Santo $J^{l}$, Araújo $F^{l}$, \\ Vinhas de Sousa $G^{l}$, Ducla Soares $J L^{l}$ \\ ${ }^{1}$ Hospital de Santa Maria, Lisbon, Portugal
}

Introduction: Systemic lupus erythematosus (SLE) is a multisystem chronic disease, witch could affect any part of the nervous system. The involvement of the central and peripheral nervous system in this autoimmune disease is well established. However, the autonomic evaluation in SLE has been rarely studied, with poor data proving the involvement of the autonomic nervous system in this pathology. None of the previous studies try to understand if there is variations in the autonomic function related with SLE flares.

Methods: We want to study a 15 SLE patients' population (according to the 1997 Diagnostic Criteria of the American College of Rheumatology). We define like exclusion criteria any disease that could affect, per se, the autonomic function and established two evaluation times for each patient (SLEDAI without exacerbation criteria and with severe flare). In each evaluation we obtained information about the disease, symptoms related with autonomic dysfunction, cardiovascular reflexes evaluation and sudomotor function, with the objective of comparing results between the two previously defined periods.

Preliminary results: We evaluated seven patients with severe flare's criteria according to SLEDAI and obtained normal age-matched values. However, none of these patients is, until now, in a remission phase to compare the results.

\section{P06}

\section{Cardiac damage and arterial stiffness in non-diabetic autonomic neuropathy}

\author{
Milazzo $V^{l}$, Di Stefano $C^{l}$, Servo $S^{2}$, Bruno $G^{l}$, Maule $S^{l}$, Veglio $F^{l}$ \\ ${ }^{1}$ Autonomic Unit and Hypertension Unit, Department of Medicine \\ and Experimental Oncology, S. Giovanni Battista Hospital, \\ University of Turin, Turin, Italy. ${ }^{2}$ Department of Neurology, \\ Maggiore della Carità Hospital, Eastern Piedmont University, \\ Novara, Italy
}

Autonomic failure (AF) is characterized by orthostatic hypotension $(\mathrm{OH})$ and supine hypertension; similarly to essential hypertension (EH), patients with AF may develop left ventricular hypertrophy. Other types of cardiac and vascular damage are still unknown in AF. Aim: Aim of the study was to evaluate left ventricular mass index (LVMI), left atrial enlargement (LAe) and volume (LAVi), ventricular remodelling (relative wall thickness, RWT), arterial stiffness (pulse wave velocity, PWV) and central hemodynamic (augmentation index, AIx) in AF.

Methods: 18 patients with non-diabetic AF (4 multiple system atrophy, 5 pure autonomic failure, 8 Parkinson's disease with AF, and 1 CIDP; age $66 \pm 13$ years, $33 \%$ females) and 70 patients with $\mathrm{EH}$ grade 1-2 matched for age, sex and body mass index, referred to our Unit from 2009 to 2011, were studied. They underwent a clinical and echocardiographic evaluation, and PWV and AIx measurements.

Results: Compared to EH, patients with AF had higher RWT $(0.44 \pm 0.08$ vs. $0.40 \pm 0.07, \mathrm{p}=0.025)$. LAVi was higher in $\mathrm{AF}$ $\left(29.77 \pm 10.37 \mathrm{cc} / \mathrm{m}^{2}\right)$ than $\mathrm{EH}\left(21.73 \pm 6.00 \mathrm{cc} / \mathrm{m}^{2}\right)(\mathrm{p}=0.001)$. Prevalence of LAe was higher in AF than $\mathrm{EH}(50 \%$ vs $17 \%$, $\mathrm{p}=0.009$ ). Compared to $\mathrm{EH}$, patients with AF had higher PWV $(9.49 \pm 1.89$ vs $8.13 \pm 2.27 \mathrm{~m} / \mathrm{s}, \quad \mathrm{p}=0.004)$ and AIx $(35.11 \pm$
$12.31 \%$ vs $30.91 \pm 20.85 \%, \mathrm{p}=0.02)$. Pulse pressure was an independent predictor of PWV $(\beta=0.664, \mathrm{p}=0.003)$. LVMI tended to be higher in AF than $\mathrm{EH}\left(110.7 \pm 36.8\right.$ vs. $92.0 \pm 23.87 \mathrm{~g} / \mathrm{m}^{2}$, $\mathrm{p}=0.053$ ).

Conclusions: Ventricular remodelling, left atrial enlargement and arterial stiffness are well represented in AF. Autonomic dysfunction, high pulse pressure and high blood pressure variability may be responsible for such cardiac and vascular alterations. In AF patients, detection and treatment of cardiovascular alterations might have short-time benefits, such as helping in the choice of treatment for $\mathrm{OH}$, allowing a more liberal therapy for $\mathrm{OH}$ during the day and preventing severe acute cardiovascular complications.

\section{P07}

\section{Cardiac sympathetic innervation is preserved in adult-onset autosomal dominant leukodystrophy}

\author{
Terlizzi R, Guaraldi P, Cason E, Capellari S, Parchi P, \\ Donadio $V$, Liguori $P$, Cortelli $R$ \\ Department of Neurological Sciences, University of Bologna, \\ Bologna, Italy
}

Aim: Adult-onset autosomal dominant leukodystrophy (ADLD) is a rare disease caused by the duplication of the nuclear lamina gene, lamin B1 (LMNB1). The common clinical feature is autonomic dysfunction followed by cerebellar and pyramidal involvement. Previous studies showed that autonomic failure was due to a selective noradrenergic defect of the peripheral component of sympathetic skin innervation. The aim of this study is to evaluate cardiac autonomic innervations in ADLD patients.

Methods: We studied two patients: a 50 years old man and his sister, a 48 years old woman, affected by familial ADLD. The ADLD diagnosis was confirmed by LMNB1 duplication. Both patients underwent a ${ }^{123}$ I-metaiodobenzylguanidine (MIBG) scintigraphy that allows the evaluation of the catecholaminergic uptake considered biomarker of post-ganglion myocardial sympathetic innervation. MIBG is an analog of guanethidine and is taken up by the postganglionic, presynaptic nerve endings. After depolarization, MIBG is released into the synaptic cleft like norepinephrine but is not metabolized. MIBG uptake has been shown to correlate with adrenergic innervation.

Results: The myocardial MIBG uptake was normal in both patients. Conclusions: The loss of post-ganglionic myocardial sympathetic fibres is a prominent feature of Lewy body dementia, Parkinson disease and pure autonomic failure. On the other hand previous studies demonstrated that autonomic failure associated to lamin B1 duplication is characterized by a selective failure of sympathetic vascular control and skin biopsy showed a selective damage of the skin efferents sympathetic fibres. This report excluding in ADLD a defect in cardiac sympathetic innervation, supports that autonomic failure resulted from an isolated damage of peripheral noradrenergic transmission that may be an useful clinical hallmark for ADLD diagnosis.

Acknowledgments: The financial support of Telethon Italy (Grant no. GGP10184 is gratefully acknowledged.

\section{References:}

1. Brown RT, et al. Adrenergic dysfunction in hereditary adult-onset leukodystrophy. Neurology. 37:1421-4.

2. Guaraldi $\mathrm{P}$, et al. Isolated noradrenergic failure in adult-onset autosomal dominant leukodystrophy. Autonomic neuroscience: basic and clinical. 2011;159:123-6.

3. Rascol O, et al. 123I-Metaiodobenzylgianidine scintigraphy in Parkinson disease and related disorders. Mov Disord. 2009;24(suppl. 2):S732-41. 
4. Treglia $\mathrm{G}$ et al. Recent developments in innervation imaging using iodine-123-metaiodobenzylguanidine scintigraphy in Lewy body diseases. Neurol Sci 2010;31:417-22.

\section{P08 \\ Characterization of GI tract's autonomic innervation in a mouse model of familial amyloid polyneuropathy}

Teixeira $D^{l}$, Saraiva $M J^{l}$

${ }^{1}$ Institute for molecular and cell Biology, Porto, Portugal

Background and aims: FAP is a fatal neurodegenerative disorder characterized by the extracellular deposition transthyretin (TTR) aggregates, particularly in the PNS. FAP initially presents with symptoms that are associated with sensory and autonomic nervous system (ANS) dysfunction. These include a loss of pain and temperature sensation in the distal limbs, impotence, gastrointestinal disturbances, bladder dysfunction and postural hypotension. As FAP progresses, sensory deficiencies extend to more proximal regions of limbs and cardiac insufficiency and mal-absorption from the gut become common.

Recently, a double-transgenic mouse line has been generated in our lab that expresses the Human mutant V30M TTR (HM30) in the absence of endogenous expression of the transcription factor, Heat shock factor 1 (Hsf1) hereafter referred to as HSFHM.

Methods: We used semi-quantitative analysis by means of immunofluorescense in order to measure both sympathetic (using tyrosine hydroxylase- $-\mathrm{TH}$ ) and parasympathetic (with vesicular acetylcholine transporter-VaChT) innervation in the GI tract of HM30 and HSFHM30 models, when compared to controls HSFiw (Wild type for TTR) and HSFM (null for TTR).

Results: Results show that at the age of 6 months GI tract sympathetic and parasympathetic innervation is not affected in either of the studied models when compared to controls; however, at the age of 12 months we observe a severe decrease in parasympathetic innervation of HSFHM30 model.

Conclusions: Given the fact that in the GI tract parasympathetic stimuli typically stimulate digestive activities, and our models present with parasympathetic defects, we believe further investigation will allow us to understand if and how the parasympathetic dysfunction is affected in FAP patients.

\section{P09}

\section{Cortical evoked responses after electrical stimulation of the bladder - a feasibility study}

\author{
Gregorini $F^{l}$, Wöllner $J^{l}$, Schubert $M^{l}$, Curt $A^{1}$, Kessler $T M^{l}$, \\ Mehnert $U^{1}$ \\ ${ }^{1}$ Uniklinik Balgrist, Universität Zürich, Zürich, Switzerland
}

Aims: Evoked potentials (EP) following electrical stimulation of defined areas within the bladder could be applied to disclose specific afferent inputs relevant to the neural control of micturition and thus may improve neurophysiological diagnostics in neurogenic bladder dysfunction. So far, EPs from the bladder have not been established to complement the urological assessment.

Methods: Electrical stimulation was applied with a special transurethral catheter to the bladder dome and trigone in seven healthy female volunteers with radiologically confirmed positioning. All subjects were measured three times. The impulses were applied with $0.5 \mathrm{~Hz}$ and with $3 \mathrm{~Hz}$. EPs were recorded at the $\mathrm{Cz}$ electrode, referenced to the $\mathrm{Fz}$ electrode, the data were filtered from $0.5-70 \mathrm{~Hz}$ and a Notch filter. Latencies and amplitudes of the EPs were determined.

Results: Following $0.5 \mathrm{~Hz}$ stimulation at the bladder dome,), a first negative peak $\mathrm{N} 1$ at $121.8 \pm 14.3 \mathrm{~ms}$ (ICC $=0.77$ ). The interpeak amplitude was $4.1 \pm 1.8 \mu \mathrm{V}$ from $\mathrm{P} 1$ to $\mathrm{N} 1$ and $5.7 \pm 2.6 \mu \mathrm{V}$ from $\mathrm{N} 1$ to $\mathrm{P} 2$. Following the trigonal stimulation, N1 latencies was $130.7 \pm 21.5 \mathrm{~ms} \quad(\mathrm{ICC}=0.62)$. The interpeak amplitude was $2.5 \pm 1.4 \mu \mathrm{V}$ from $\mathrm{P} 1$ to $\mathrm{N} 1$ and $5.2 \pm 2.1 \mu \mathrm{V}$ from $\mathrm{N} 1$ to $\mathrm{P} 2$.

For the $3 \mathrm{~Hz}$ stimulation, no reliable responses could be recorded.

Conclusions: The $0.5 \mathrm{~Hz}$ stimulation of the trigone and dome of the human bladder generates reproducible EPs. The sensory thresholds, late latencies and the lack of responses with $3 \mathrm{~Hz}$ stimulation indicate that the afferent input is probably conducted by less myelinated fibres (i.e. A-delta fibers). Depending on further confirmation and studies in patients, the data reveal that specific A-delta EP of the bladder might become feasible for diagnostic purposes.

\section{P10}

Cutaneous sympathetic activity was reduced in patients with complex regional pain syndrome type 1

\author{
Asahina $M^{l}$, Poudel $A^{l}$, Fujinuma $Y^{l}$, Katagiri $A^{l}$, Yamanaka $Y^{l}$, \\ Akaogi $Y^{1}$, Kuwabara $S^{1}$ \\ ${ }^{1}$ Chiba University School of Medicine, Chiba, Japan
}

Aim: The aim of this study was to see whether sympathetic vasoconstrictor and sudomotor function are altered in patients with complex regional pain syndrome type 1 (CRPS I).

Methods: We studied 10 CRPS I patients $(40.5 \pm 12.9$ years, disease duration $20 \pm 22$ months) and 20 normal healthy subjects (age $42.2 \pm 13.8$ years). All the CRPS I patients had pain in the unilateral upper limb. In each participant sympathetic sweat responses (SSwRs) were measured on the thumb pad in the affected limb by a ventilated capsule sudorometer (SK-1000, Skinos) and skin vasomotor reflexes (SkVRs) were recorded on the index finger pad in the affected limb by Doppler flow meter (ALF 210, Advance) during deep inspiration, mental arithmetic and isotonic exercise.

Results: There were no significant differences in baseline values of skin blood flow and sweat output between two groups. Although all the controls showed SkVRs and SSwRs, one CRPS I patients did not present SkVRs, and four patients did not SSwRs for any procedures. Our CRPS I patients showed significantly lower SkVR amplitudes than the control group for deep inspiration $(\mathrm{p}=0.04)$ and isotonic exercise $(p=0.01)$. There were no significant differences in mean SSwR amplitudes for any stimuli between two groups. There were no significant correlations between duration and SkVRs or SSwRs.

Conclusion: Our results revealed reduced skin sympathetic activities in CRPS I. Some researcher have speculated that CRPS is a posttraumatic neuralgia associated with distal degeneration of smalldiameter peripheral axons. Our results may reflect underlying small fiber involvement in CRPS I.

\section{P11}

\section{Does the intradetrusor application of botulinumtoxin A affect the heart rate variability?}

Wöllner $J^{l}$, Gregorini $F^{l}$, Kozomara $M^{l}$, Kessler TM $M^{l}$, Mehnert $U^{l}$ ${ }^{1}$ Neuro-Urology, Spinal Cord Injury Center, University of Zürich, Balgrist University Hospital, Zürich, Switzerland 
Aim: Heart rate variability (HRV) is an established method to monitor the function and integrity of the autonomous nerve system (ANS).The BoNT/A application in the detrusor muscle is applied in neurogenic-and non-neurogenic detrusor overactivity, to improve the bladder function. Aim of the study, was to evaluate a potential impact on the HRV following BoNT/A injection, as a systemic side effect. Methods: In four visits (1-4), a three channel ECG (10 min) was recorded in a resting, supine position. The treatment group received BoNT/A injection between visit 2 and 3. Medication with impact on the HRV and/or diseases of the cardiovascular system were an exclusion criteria. Recordings and analysis of the frequency and time domain parameters were performed with Soleasy ${ }^{\circledR}$ software (Alea solutions;Switzerland) All values were log 10 transformed and given as mean and standard deviation. Due to multiple comparisons, $\alpha$ was corrected to 0.0125 (Bonferroni method).

Results: 24 patients were included, 12 with neurogenic bladder

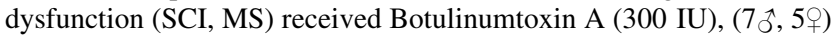
mean age: 45.6 years $( \pm 13.5)$. The remaining were controls, $(8 \hat{0}, 4$ 우), mean age: 43.2 years $( \pm 11.1)$ without treatment. Comparing both groups during the four subsequent visits, no differences in time- and frequency domain parameters occured, VLF (2.66 vs. 2.90), LF (2.51 vs. 2.77$), \mathrm{HF}$ (2.27 vs. 2.51 ), the LF/HF ratio (1.15 vs. 1.13$)$, RMSSD (1.49 vs. 1.56) and SDNN (1.65 vs. 1.70) do not reveal significant differences before and after the BoNT/A application.

Conclusions: To our knowledge, this is the first study investigating potential effects on the HRV, following intradetrusor injection of BoNT/A due to neurogenic detrusor overactivity. Applying these technique no relevant changes in HRV occur neither immediate after the application nor in the follow-up 6 weeks later.

\section{P12}

\section{Effects of $0.1 \mathrm{~Hz}$ breathing and acute mental stresss on peripheral vascular activity in physically trained and sedentary young healthy adults}

\author{
Potocnik $N^{1}$, Cankar $K^{1}$, Melik $Z^{1}$, Starc $V^{1}$ \\ ${ }^{1}$ University of Ljubljana, Medical faculty, Institute of physiology, \\ Ljubljana, Slovenia
}

Favourable effects of aerobic training on autonomic balance and baroreflex function have been reported, but its beneficial effects on the vascular function of healthy subjects have not been proven yet. Aim: The aim of our study was to obtain the ability of modulation of peripheral arterial compliance provoked by different autonomic stimuli $(0.1 \mathrm{~Hz}$ breathing and mental stress) in two groups of healthy young adults: physically trained (group A) and sedentary controls (group B).

Methods: Experiments were performed on 21 subjects, 19-24 years old (12 in group A, 11 in group B). We measured ECG, arterial blood pressure and finger artery compliance at rest, 3 min during paced $0.1 \mathrm{~Hz}$ breathing and $3 \mathrm{~min}$ during mental stress (standard arithmetic challenge). A noninvasive method was used to measure finger artery compliance, compliance index (CI) was calculated. Baroreflex sensitivity (BRS) was determined using the sequential method.

Results: Our results revealed the elevated CI in group A compared to the group B $(3.42 \pm 0.30$ and $1.28 \pm 0.31, p=0.004$, t test $)$ at rest, but no significant differences in $\mathrm{CI}$ between groups during both physiological stimuli. CI decreased during $0.1 \mathrm{~Hz}$ breathing $(1.53 \pm$ $0.20, \mathrm{p}=0.003$, paired $\mathrm{t}$ test $)$ and mental stress $(0.87 \pm 0.13$, $\mathrm{p}=0.002$, paired $\mathrm{t}$ test) in group $\mathrm{A}$, but only during mental stress in group B $(0.59 \pm 0.12, p=0.03$, paired t test $)$. BRS did not differ between groups, but was significantly reduced in both groups during mental stress (from $20.9 \pm 3.3$ to $16.2 \pm 2.2$ in group A and from
$25.1 \pm 3.8$ to $16.3 \pm 1.4$ in group $\mathrm{B}, \mathrm{p}=0.004$, paired $\mathrm{t}$ test). There were no changes in BRS during $0.1 \mathrm{~Hz}$ breathing $(22.3 \pm 3.3$ in group A and $26.4 \pm 3.1$ in group B).

Conclusions: Regular aerobic exercise increases peripheral arterial compliance in healthy subjects. Surprisingly the increase was not associated with a greater BRS indicating that peripheral and not central autonomic mechanisms govern peripheral arterial properties.

\section{P13}

\section{Functional magnetic resonance imaging of central nervous system autonomic during sympathetic and parasympathetic stimulation}

Aguiar $P^{l}$, Lauterbach $M^{l}$, Reimão $S^{l}$, Ducla Soares $J L^{1}$

${ }^{1}$ Hospital de Santa Maria, Lisbon, Portugal

Introduction: Currently, the clinical evaluation of the autonomic nervous system dysfunction is based, mainly, in the analysis of cardiovascular reactivity and heart rate variability. There are few studies concerning the functional evaluation of the Central Nervous Autonomic System in response to the classical stimulatory or inhibitory maneuvers. Aims: The goal of our research is to compare the brain activation pattern during classical excitatory or inhibitory sympathetic or parasympathetic maneuvers in three groups of subjects, in order to identify changes in brain activation by functional MRI .

Methods: The three population groups are: 30 healthy people (10 of them required for a pilot study), 20 patients with clinically diagnosed central autonomic failure and 20 patients with peripheral autonomic failure. Out of the scanner all participants will undergo the following evaluation: clinical protocol (heat-up tilt test, handgrip, Valsalva maneuver, deep breathing, inspiration gap, cold pressor, short term HR variability analysis) and imaging protocol (long and short thermal stimulation, hyperventilation and inspiratory capacity apnoea). Beside the classical statistical approach with the General Linear Model the data will be analyzed by alternative data-driven, e.g. the independent component analysis.

Preliminary results/data analysis: In a pilot study with three controls and one patient, similar activation patterns to those reported in previous studies were obtained. In order to identify the physiological neuronal circuit of the autonomous system, a group analysis of the healthy controls will be carried out. The activation patterns of the pathological cases will then be compared to that standard. In a final step, patient data will be grouped and compared according to the etiology of the autonomous system dysfunction (central or peripheral).

\section{P14}

Glucoregulation and autonomic function in older male patients with diabetes mellitus

Gilden $J^{1}$, Cheng $J^{1}$, Theckedath $B^{2}$, Hung $P^{l}$, Farahani $M^{1}$, Azul $J^{1}$, Kesari $N^{l}$, Shankar $S^{l}$, Kandavel $P^{I}$

${ }^{1}$ Rosalind Franklin University of Medicine and Science/Chicago Medical School, North Chicago,IL, USA. ${ }^{2}$ James A. Lovell Federal Health Care Center, North Chicago, IL, USA

Aims: Glycemic control is important for prevention of chronic complications in diabetes mellitus (DM). However, recent studies, such as ACCORD and ADVANCE, have suggested that the risk of mortality is higher in those patients with extremely tight glucose control, especially with longer duration of diabetes and already established chronic complications. Others have suggested that the 
increased risks of hypoglycemia (HYPOS) may be responsible for further autonomic failure (AN) and sudden death. We evaluated whether glucose regulation is altered by AN in older male DM.

Methods: 31 male DM [(8 Type 1: 23 Type 2) (age $=63.6 \pm$ 1.3 years) (duration $\mathrm{DM}=18 \pm 1.3$ years) $\quad(\mathrm{BMI}=33.3 \pm 0.9$ ) $($ Hgbalc $=7.95 \pm 0.17 \%) \quad($ significant peripheral neuropathy by monofilament and urine microalbumin/Cr ratio $(\mathrm{MA})=149 \pm 81.8$ ) (Bazett QTC $=401-464 \mathrm{~mm}$ )], were identified by retrospective chart review of unbiased glucose assessments by $72 \mathrm{~h}$ Continuous glucose monitoring system (CGMS). Glucose values were collected every 5 min., mathematically transformed and expressed as $\%$ of time above normal (>140 mg\%) (\%AN), \% normal (70-140 mg\%) (\%N), and $\%$ below normal $(<70 \mathrm{mg} \%)(\% \mathrm{BN})$ for 3 time intervals: (T1 $=0600-1800$ hours $) ; \quad(\mathrm{T} 2=1800-2400$ hours $) ; \quad(\mathrm{T} 3=2400-$ 0600 hours). Glucose averages for $24 \mathrm{~h}$ time period were also calculated (\%TAN, \% TN, and \%TBN). DM were divided into two groups by QTC $<450(\mathrm{n}=18)$ versus QTC $\geq 450(\mathrm{n}=13)$.

Results: Despite similar Hgbalc, DM with QTC $<450$ had more HYPOS (overall \% TBN $=5 \pm 1.4$ vs. $2.3 \pm 0.7$ ), with $\mathrm{T} 1=3.8 \pm$ 1.2 vs. $2.3 \pm 1.1 \%$ ); $<<0.05$ ). For T2, only 7:18 QTC $<450$ had HYPOS $(14.4 \pm 3.5 \%)$ versus $6: 13$ for QTC $\geq 450(4.7 \pm 1.9 \%)$ and T3 $(11.9 \pm 2.3$ for $10: 18$ QTC $<450$ vs. $5.2 \pm 1.7 \%$ for $5: 13$ QTC $\geq 450 ; \mathrm{p}<0.02$ ).

Conclusions: Although Hgba1c was similar in both groups, specific time periods were more likely to be vulnerable to hypoglycemia. These responses were dependent upon the integrity of the autonomic nervous system. Therefore, it is important to evaluate autonomic function when recommending a regimen of tight glycemic control in older male patients with diabetes mellitus.

Acknowledgments: Research sponsored by JAL FHCC.

\section{P15}

\section{Heart rate modulation of vocal acoustics. Physiological mechanisms}

\author{
Dawid-Milner $M S^{1}$, Alguacil $M D^{2}$, Barón-López $F J^{3}$, \\ Reyes-Bueno $\mathrm{JA}^{1}$, Bermúdez de Alvear $\mathrm{RM}^{4}$ \\ ${ }^{1}$ Unidad de Neurofisiología del Sistema Nervioso Autónomo \\ (CIMES); Universidad de Málaga, Málaga, Spain. ${ }^{2}$ Servicio de \\ Otorrinolaringología; Hospital Regional Universitario Carlos Haya., \\ Málaga, Spain. ${ }^{3}$ Departamento de Medicina Preventiva y Salud \\ Pública; Facultad de Medicina; Universidad de Málaga, Málaga, \\ Spain. ${ }^{4}$ Departamento de Radiología, Oftalmología y \\ Otorrinolaringología; Facultad de Medicina; Universidad de Málaga, \\ Málaga, Spain
}

Orlikoff(1) showed that heartbeat modulates fundamental frequency (Fo) of human voice. This effect appears to be related, among others, to cardiovascular (CV) changes induced in vocal-fold biomechanics and to heartbeat modulation of subglottal pressure during the heart cycle. However, it is still unclear how heart rate (HR) changes modify human voice and the relations between $\mathrm{CV}$ and vocal acoustic parameters.

Fourteen healthy volunteers ( 7 females and 7 males, mean age $22 \pm 1.3$ years) were included in this study. None of these subjects had clinical signs of $\mathrm{CV}$, neurological or metabolic disorders, and none was under medication. Arterial blood pressure (ABP), ECG and glottal sound were continuously monitored. Instantaneous HR, systolic, diastolic, and mean ABP changes were analysed during three autonomic tasks; the isometric, cutaneous cold and mental arithmetic tests. To obtain the voice samples, subjects were asked to sustain the Spanish/æ/vowel for $5 \mathrm{~s}$; five vocal emissions were recorded at 1-min intervals before the autonomic tasks. Each autonomic task was composed of three phases (pre-test, test, and post-test), and three consecutive vocal emissions were obtained at 1-min intervals during each of these phases. KAY Elemetrics Multidimensional Voice Profile was used to analyse vocal acoustic variables.

Regression analysis of the 448 vocal emissions showed that Fo was significantly related to the HR that occurred just before each vocal emission $\left(r^{2}=0.09, p<0.001\right)$. Additionally, during the sustained vowels an increase in HR was also observed which was significantly associated to the Fo values $\left(r^{2}=0.08, p<0.001\right)$.

These results show that heartbeat variations have a regular and significant influence on phonatory frequency. Moreover, Fo and CV parameters are consistently associated during control conditions as well as during autonomic tasks. These vegetative-acoustic relations seem to reflect a specific profile in normal young subjects, independently of gender. Such systematic modulation is discussed on the basis of blood pressure related changes and blood volume-related modulation of subglottal pressure.

\section{Reference:}

1. Orlikoff RF, Baken RJ. Fundamental frequency modulation of the human voice by the heartbeat: Preliminary results and possible mechanisms. J Acoust Soc Am. 1989;85(2):888-93.

\section{P16}

\section{Late onset of autonomic symptoms in amyloidosis: a case report}

Servo $C^{l}$, Civardi $C^{l}$, Mazzini $L^{l}$, Nasuelli $N^{l}$, Milazzo $V^{2}$, Di Stefano $C^{2}$, Maule $S^{2}$, Monaco $F^{1}$

${ }^{1}$ Department of Neurology, Maggiore della Carità Hospital, Eastern Piedmont University, NOVARA, Italy. ${ }^{2}$ Autonomic and Hypertension Centre, Department of Medicine and Experimental Oncology, University of Turin, Turin, Italy

Amyloidosis is a rare disorder, characterized by extracellular deposition of abnormal proteins. Symptomatic peripheral neuropathy and autonomic failure are common findings in this disease.

We report a case of a 61-years-old male referred to our Unit in January 2010, complaining of weight loss, fatigue and painful paraesthesias, especially in lower limbs. The patient had a history of axonal sensory-motor polyneuropathy, started in 2007 and treated with steroids and immunoglobulin without remission of the symptoms. The neurological examination was normal, except for moderate hypotrophy and reduced tendon reflexes. ECG, blood and LCS exam, head MRI and total body CT were normal.

During the following months, the patient started suffering from orthostatic intolerance with "coat-hanger" back pain, postprandial hypotension, nausea, constipation or diarrhoea, urinary retention, and sexual dysfunction.

The extent and severity of autonomic failure was quantified by using a standardized battery of autonomic tests, that resulted in a severe autonomic dysfunction with symptomatic orthostatic hypotension. Therapy with non-pharmacological measures, acarbose and fludrocortisone was started.

The diagnosis of amyloidosis was made through biopsy of fat tissue. Secondary causes of amyloidosis were excluded.

Polyneuropathy may be the earliest presentation of amyloidosis, often followed by the development of autonomic symptoms after a variable time interval. Autonomic nerve fibers may be selectively or disproportionately affected. Precocious testing for autonomic neuropathy may be helpful when the diagnosis is uncertain, for example in patients who have peripheral neuropathy of unknown origin. Autonomic tests may, in fact, be abnormal before clinical autonomic symptoms begin, leading to earlier diagnosis and treatment of amyloidosis. 


\section{P17}

\section{Long term ambulatory norepinephrine infusions: a successful way of treating pure autonomic failure}

\author{
Zekeridou $A^{l}$, Michel ${ }^{l}$, Kuntzer $T^{l}$ \\ ${ }^{1}$ University Hospital of Lausanne, Lausanne, Switzerland
}

Pure autonomic failure (PAF), a degenerative disorder of the autonomic nervous system, is characterized by progressive disabling orthostatic hypotension $(\mathrm{OH})$. We here present a 48 year-old PAF patient who was successfully treated with norepinephrine pump infusions.

The patient presented with sexual dysfunction, decreased physical endurance and presented several episodes of syncopes. Neurological examination was normal except $\mathrm{OH}$. Repeated work-ups demonstrated: severe $\mathrm{OH}$ during tilt-table tests with continuous recordings of arterial blood pressures (BP); absent sympathetic skin responses in his soles; reduced $\mathrm{R}-\mathrm{R}$ heart variation rate; abnormal plasma concentrations of norepinephrine and antidiuretic hormones; decreased MIBG cardiac scintigraphy. Nerve biopsy and extended lab work, including lumbar puncture were normal.

Treatments with non-pharmacological and pharmacological measures (midodrine up to $40 \mathrm{mg} /$ day and fludrocortisone up to $0.8 \mathrm{mg} /$ day) were successful the first 12 months but they lost their efficiency afterwards and the patient had to stay lying at home. A pump for intravenous norepinephrine infusions was administered and the patient had a significant improvement with the disappearance of syncopes, started working again and was able to stop all other medication. The infusion rate needed to be adjusted twice in the following 8 years of follow up but patient continued to have an almost normal life. Cardiac follow up showed no signs of ischemia, but unfortunately patient died unexpectedly and no autopsy was performed.

To our knowledge, he is the first patient described with such a long lasting norepinephrine treatment which stays beneficial even doses had to be increased. This underscores that norepinephrine pump infusions is an useful way to treat progressive disabling $\mathrm{OH}$.

\section{P18}

\section{Natural history of non-diabetic neurogenic orthostatic hypotension: a study in an Italian cohort}

\footnotetext{
Maule $S^{l}$, Milazzo $V^{l}$, Di Stefano $C^{l}$, Servo $S^{2}$, Burrello $J^{l}$, Veglio $F^{l}$ ${ }^{1}$ Autonomic Unit and Hypertension Unit, S. Giovanni Battista Hospital, University of Turin, Turin, Italy. ${ }^{2}$ Department of Neurology, Maggiore della Carità Hospital, Eastern Piedmont University, Novara, Italy

Neurogenic orthostatic hypotension (NOH) is an invalidating condition, often confining patients to bed. The prognosis and causes of death in patients with $\mathrm{NOH}$ are likely to depend on the underlying type of autonomic neuropathy. With the exception of multiple system atrophy, the natural history of $\mathrm{NOH}$ has been poorly studied.

Aim: To evaluate retrospectively the prevalence of comorbidities, the development of cardiac complications, and to examine the causes of death of a cohort of patients with a diagnosis of non-diabetic $\mathrm{NOH}$ through a follow-up of nearly 5 years

Methods: Clinical information and causes of death were obtained from 104 patients (63 males; age 71, SD10; 45 multiple system atrophy [MSA], 9 pure autonomic failure [PAF], 43 Parkinson's disease with autonomic neuropathy [PD], and 7 other types),
}

referred to the Autonomic Unit from January 1, 1996 to December 31, 2009.

Results: Hypertension was present in $31 \%$, atrial fibrillation in $4.8 \%$, and heart failure in $21.2 \%$. At the end of follow-up, 44 patients were deceased $(62 \%$ MSA, 30\% PD, 22\% PAF, and 14\% other types, $\mathrm{p}=0.0001)$. Type of NOH (HR 0.16, CI $0.03-0.82, \mathrm{p}=0.02)$ and hypertension (HR 2.33, CI $0.93-5.81, \mathrm{p}=0.07$ ) were the main factors associated to mortality. Causes of death were infective $(34.1 \%)$, respiratory $(20.5 \%)$, and cardiac $(15.9 \%)$. NOH had a threefold increased risk of mortality with respect to the general Piedmont population. The relative risk was particularly high for death from cachexia (standardized mortality ratio, SMR 21.9) infections (SMR 141.8), and respiratory diseases (SMR 7.5).

Conclusions: Type of $\mathrm{NOH}$ and hypertension represent the main factors conditioning outcome. In MSA, mortality is mainly due to infective and respiratory causes. In the other types, bearing a better prognosis, the weight of cardiac complications might be more relevant.

\section{P19}

\section{Outcome of head-up tilt test affected by tilting speed}

Kirbiš $M^{3}$, Meglič $B^{1}$, Grad $A^{4}$, Bajrović $F F^{2}$

${ }^{1}$ University Medical Centre Ljubljana, Ljubljana, Slovenia. ${ }^{2}$ Medical Faculty, University of Ljubljana, Ljubljana, Slovenia. ${ }^{3}$ General hospital Nova Gorica, Šmpeter pri Gorici, Slovenia. ${ }^{4}$ Isola General Hospital, Izola, Slovenia

Aims: To test the hypotheses of possible influence of tilting speed and arm position on outcome of head-up tilt (HUT) from our recent retrospective analyses 1 we performed a prospective study separately analysing each of these two variables.

Methods: Two groups of patients underwent a set of two $60^{\circ} \mathrm{HUT}$ tests; the first test was always a short, 20-min passive HUT test, the second was a long, 45-min HUT test with glyceryl trinitrate provocation. In group one $(\mathrm{n}=100)$ we compared slow tilting-up (15 s, $4 \%$ ) and fast tilting-up ( $2 \mathrm{~s}, 30 \%$ ), always with arm supported at heart level; one half of patients had the short test done with slow tilting and the long test with fast tilting, the other half had the reversed order of tilting speed. In group two $(n=40)$ one half had the short test done with arm hanging down and the long test with arm outstretched at heart level; the other half had reversed order of arm positions. Blood pressure (BP), heart rate (HR) and outcome of HUT test were compared between tilting speeds and between arm positions.

Results: Syncope was induced in $38(76 \%)$ subjects after slow tiltingup and $30(60 \%)$ subjects after fast tilting-up $(\mathrm{p}=0.10)$. Slower tilting-up was associated with higher HR increase at $3 \mathrm{~min}$ of HUT (15.9 and 11.8 beats/min after slow and fast tilting, respectively, $\mathrm{p}<0.05)$. BP changes were similar after both tilting speeds. There was no difference in HR, BP or rate of syncope induction between arm positions.

Conclusion: Although the increase in the rate of syncope induction after lowering the tilting speed did not reach statistical significance, our results support the hypothesis that hemodynamic response to HUT depends on tilting speed. Clarification of the mechanism behind this finding warrants further studies.

\section{References:}

1. Kirbiš M, Meglič B, Grad A, Bajrović FF. Unexplained changes in tilt table test outcomes after moving to a new laboratory. Clin Auton Res 2010;20:385-6. 


\section{P20}

\section{Peripheral neuropathy in patient with multiple myeloma treated with bortezomib}

\author{
Aguiar $P^{l}$, Cruz $D^{I}$, Ferro Rodrigues $R^{l}$, Fajardo $J^{1}$, Araújo $F^{l}$, \\ Ducla Soares $J L^{l}$ \\ ${ }^{1}$ Hospital de Santa Maria, Lisbon, Portugal
}

Introduction: The presence of peripheral neuropathy symptoms in a patient with multiple myeloma (MM) implies an investigation regarding the mechanisms of the disease. Symptoms could be related with the disease itself or iatrogenic effects of novel therapeutics, like bortezomib.

Case report: A 58 year woman diagnosed with MM in March 2009 was teated with radiotherapy to an occipital plasmocytoma and started quemotherapy (QT) with bortezomib, dexamethasone and doxorubicin with complete hematologic response. Distal paresthesias soon developed, worsening during the third QT cycle (with extension to tight and shoulders). Severe orthostatic hypotension, with recurrent syncope maintained the patient bed ridden for several weeks. She performed electromyography (severe sensitive axonal neuropathy, with autonomic compromise), tilt table testing (orthostatic hypotension), spinal cord MRI (involvement by MM, without cord injury), sural nerve biopsy (toxic neuropathy) and normal abdominal fat aspirate, echocardiogram, renal ultrasound and cortisone levels.

Iatrogenic neuropathy was diagnosed and therapy with fludrocortisone, midodrine and gabapenthine prescribed, without improvement. Thus, a 4 days cycle of intravenous immunoglobulin (IVIg) improved the symptoms and orthostatic hypotension disappeared.

Discussion: Bortezomib is a first line treatment to MM. The association between this therapy and neuropathy has been described in about $35 \%$ of the patients, with variable severity. However, the singularity of this case is related with the severe autonomic involvement (very rarely described in the literature) and the response to IVIg.

\section{P21}

\section{Postural Tachycardia Syndrome: how much "sustained" is the heart rate increment?}

Calandra-Buonaura $G^{l}$, Guaraldi $P^{l}$, Corazza $I^{2}$, Cece $E^{l}$, Solieri $L^{l}$, Barletta $G^{I}$, Cortelli $P^{I}$

${ }^{1}$ Dipartimento di Scienze Neurologiche, Università di Bologna, Bologna, Italy. ${ }^{2}$ Dipartimento Cardiovascolare, Università di Bologna, Bologna, Italy

Aim: According to the recent Consensus (Freeman et al. 2011), Postural Tachycardia Syndrome (PoTS) is characterized by a "sustained" heart rate (HR) increment $\geq 30$ beats/min ( $\geq 40$ beats/min for individual aged 12-19 years) within $10 \mathrm{~min}$ of standing or head-up tilt (HUT) in the absence of orthostatic hypotension. Aim of the present study was to quantify the duration of the HR increment during HUT in a cohort of PoTS patients

Methods: 34 consecutive patients ( 24 females; mean age $25.1 \pm 9.1$ years, range 13-43), with a history of orthostatic intolerance or syncope, diagnosed as PoTS in our Department after performing HUT with continuous blood pressure (BP) and HR monitoring (Task Force Monitor or Portapres model II), were included in the study. RR intervals and BP values were resampled off-line at $4 \mathrm{~Hz}$. The mean BP and HR values from the last $5 \mathrm{~min}$ preceding the HUT were considered as baseline. The percentage of the first $10 \mathrm{~min}$ of HUT during which the HR increment fulfilled the criteria for PoTS $\left(\% \_30=\geq 30\right.$ beats $/ \mathrm{min}$; $\% \_40=\geq 40$ beats $/ \mathrm{min}$ ) was calculated.
Results: Among the 21 patients aged over 19, mean \%_30 was $51.4 \pm 30.5 \%$ (range $5.2-97.5 \%$ ). In 11 patients \%_30 was $\geq 50 \%$, in five patients it was comprised between 20 and $50 \%$, in three between 10 and $20 \%$ and in the last two it was $<10 \%$. Among the 13 patients aged 12-19 years, mean \%_40 was $42.1 \pm 23.9 \%$, ranging from 6.7 to $85.3 \%$. 14 patients complained of symptoms typical of PoTS, whose occurrence did not correlate with HR increment duration. Conclusions: Our study demonstrated that PoTS patients display a heterogeneous HR increment during HUT, which did not correlate with symptoms' occurrence.

Reference: Freeman R et al. Clin Auton Res 2011;21:69.

\section{P22}

Pulse rate and mean blood pressure variability during the head-up tilt table test in high temperature before and after a short exercise-heat acclimation period

Jagomagi $K^{1}$, Talts $J^{l}$, Raamat $R^{l}$, Ates $O^{3}$, Karelson $K^{1}$, Burk $A^{l}$, Ö̈pik $V^{1}$, Cotuk $H B^{4}$

${ }^{1}$ Department of Physiology, University of Tartu, Tartu, Estonia.

${ }^{2}$ Institute of Exercise Biology and Physiotherapy, University of Tartu, Tartu, Estonia. ${ }^{3}$ Istanbul University, Istanbul, Turkey. ${ }^{4}$ Marmara University, Istanbul, Turkey

The aim of the present study was to evaluate changes in mean blood pressure (MBP) and pulse rate (PR) during head up tilting (HUT) in hot conditions before and after a 8-day exercise-heat acclimation program.

Methods: 18 healthy male volunteers participated in the study, their mean age, height, and weight were $23.4 \pm 3.2$ years, $181.1 \pm 5 \mathrm{~cm}$ and $80.2 \pm 11.2 \mathrm{~kg}$, respectively. HUT was performed in climate chamber $\left(42^{\circ} \mathrm{C}\right.$, relative humidity $\left.18 \%\right)$ before and after a 8 -day exercise-heat acclimation program. The HUT protocol comprised three 10-min lasting phases: (1) supine baseline, (2) tilting at $70^{\circ}$ and (3) resupine. Beat-to-beat non-invasive mean blood pressure and pulse rate were continuously recorded by physiograph. Frequency domain analysis was performed during the last $5 \mathrm{~min}$ of baseline and tilting.

Results: No symptoms and signs of syncope in studied volunteers were detected during tilting. Mean PR was lower in post-acclimation test (58.6 vs. $65.5 \mathrm{bpm}$ in supine; 80.1 vs. $88.2 \mathrm{bpm}$ in tilting position). LF/HF ratio of pulse rate (the quantitative index of the sympatovagal balance) was slightly increased in post-acclimation test ( 2.29 vs. 1.88 in supine; 4.11 vs. 3.69 in tilting position). The absolute power of the LF component of MBP (assumed as index of sympathetic activity) was similar for post- and preacclimation test in supine position $\left(9.4\right.$ vs. $\left.9.5 \mathrm{mmHg}^{2}\right)$ and slightly increased in tilting position (15.7 vs. $\left.14.6 \mathrm{mmHg}^{2}\right)$.

Conclusion: A 8-day exercise-heat acclimation program resulted in a decrease of pulse rate. At the same time no significant changes in spectral indices of PR and MBP variability were observed.

Acknowledgments: This study was supported by Estonian Science Foundation (Grants 6947 and 7723) and Estonian Ministry of Education and Research (SF0180148s08).

\section{P23}

\section{Relationship between orthostatic hypotension and cognitive impairment in Parkinson disease}

Di Leo $R^{l}$, Biundo $R^{l}$, Gasparoli $E^{l}$, Pilleri $M^{l}$, Facchini $S^{l}$, Bernardi $L^{l}$, Antonini $A^{l}$

${ }^{1}$ IRCCS San Camillo, Venice, Italy 
Background: Autonomic dysfunction is frequent in patients with parkinsonism associated with dementia like Parkinson disease dementia (PDD) and Lewy body disease (LBD), while in Parkinson disease (PD) it is less frequent and appears in the late stages of the disease.

Exploring the relationship between autonomic dysfunction and the presence and severity of cognitive decline is crucial to understand the mechanisms underlying the progression of the disease and the expression of clinical features of PD.

Objective: The aim of the present study is to assess the prevalence and severity of cognitive impairment in PD patients with and without orthostatic hypotension $(\mathrm{OH})$.

Materials and methods: We studied 32 consecutive PD patients. Patients with clinical features suggesting a diagnosis of PDD and LBD were not included.

Presence of $\mathrm{OH}$ was assessed with a tilt test (TT).

TT was considered abnormal when there was a sustained reduction of systolic blood pressure of at least $20 \mathrm{mmHg}$ or diastolic blood pressure of $10 \mathrm{mmHg}$ within $3 \mathrm{~min}$ of head up tilt to $60^{\circ}$.

All patients underwent a neuropsychological assessment exploring executive function, attention, memory and visuo-spatial deficits. Severity of motor symptoms was evaluated by means of the motor part MDS-UPDRS.

Results: Sixteen patients presented $\mathrm{OH}$, while 16 did not. Patients with $\mathrm{OH}$ did not show significant differences in any neuropsychological tests compared with patients without $\mathrm{OH}$.

Discussion: Our study did not demonstrate any correlation between cardiovascular autonomic dysfunction and cognitive impairment in patients with PD without dementia. Although a higher prevalence of autonomic dysfunction has been reported in PDD and LBD, it seems that in PD, orthostatic hypotension and cognitive decline are two independent non motor symptoms, whose expression and progression may be unrelated.

\section{P24}

\section{Syncope induced by multiple factors}

Wu $C L^{l}$, Ho $W M^{l}$, Weng $W C^{l}$, Huang $W Y^{l}$, Chien $Y Y^{l}$, Peng $T I^{l}$ ${ }^{1}$ Department of Neurology, Chang-Gung Memorial Hospital at Keelung, Keelung, Taiwan. ${ }^{2}$ School of Medicine, Chang-Gung University, Tauyuan, Taiwan

Aim: Differential diagnosis of syncope has always been a challenge to Neurologist and Cardiologist, many times a definite diagnosis could not be made even extensive studies had been done. We present a case of syncope and discuss the combined neurogenic, vasogenic, and metabolic inducing factors.

A 75 year-old man with history of hypertension and hyperlipidemia developed three attacks of syncope within 1 year, twice during cleaning the house in the morning, and once during taking a bath. After detail autonomic, cardiologic, metabolic, and images studies, including tilting table, Valsava maneuver, continuous blood pressure monitoring, R-R interval, transcranial doppler, electroencephalography, cardiac echo, 24-h Holter monitor, and MR angiography, there is no one single risk factors could explain or reproduce the attack. Multiple factors, including excessive anti-hypertensive medications, dysautonomia, vertebrobasilar insufficiency, and possible poor hydration induced relative hypovolemia were concluded. After correction of these factors the outcome was good.

Conclusions: Syncope may not be developed only due to a single factor because the brain blood flow could be maintained by multiple compensating hemodynamic mechanisms. In aged peoples with multiple metabolic disorders, dysautonomic neurogenic and vasogenic risk factors may be combined. In our case, the dose and timing for daily anti-hypertensive medications, treatment of dysautonomia, and well hydration are all necessary. In such aged cases, multidisciplinary approach and management are essential.

References:

1. Mathias CJ. Clin Auto Res. 2004;14:(Supplement 1): i45.

2. Moya A et al. Eur Heart J. 2009;30:2631.

3. Britton J et al. Clin Auto Res. 2004;14:148.

4. Mathias CJ. Cardiovasc Med. 2007;8:1883.

5. Brignole M. Heart. 2007;93:130.

6. Thijs RD et al. Clinic Auto Res. 2010;20:19.

7. Chen LY, et al. Mayo Clin Proc. 2008;83:1280.

P25 The genetically determination of autonomic nervous system and new approach in the treatment of syncope and orthostatic intolerance using homeopathic phorm of drugs

Milovanovic BM ${ }^{I}$, Radivojevic $V R^{I}$, Mutavdzin $S M^{I}$, Krajnovic $Y K^{1}$, Kosa $B^{l}$

${ }^{1}$ Neurocardiological Laboratory,University Clinical Center, Belgrade, Serbia and Montenegro

In order to precisely define the effect drugs on sympathovagal balance we hypothesized that the effects of the drug depend greatly on the basal state of sympathovagal balance or sympathal or vagal predominance. Having in mind that basal sympathovagal balance differs among patients, in the first phase of our study we determined the type of disturbance, that is, dominance in patients with syncope,orthostatic hypotension and hypotension. In order to thoroughly defined genetically impact on autonomic nervous system function we analyzed the sympathovagal balance using autonomic nervous system activity (ANSA) method. In order to reveal the right mechanisms of syncopal atacks we developed Ansa Scan software using analysis of spectral parameters as a main markers of simpatho-vagal balance. Low frequency (LF) (nu) corresponding with sympathetic activity and high frequency $(\mathrm{HF})(\mathrm{nu})$, corresponding with parasympathetic activity were parameters of short-term beat-to-beat spectral HRV analysis used in ANSA method. Using normal cut of values for these parameters, we divided our patients into two main groups depending whether LF (nu) or HF (nu) predominated: (1) patient with high sympathetic activity (sympathetic group) and (2) patients with high vagal activity (parasympathetic group). Patients were also divided in subgroups related to stage of vagal of sympathetic predominance(high or moderate). The final conclusion was that patients with orthostatic intolerance are with vagal pattern of disfunction with lack of sympathetic function. In order to improve sympathetic function we treated three patients with orthostatic hypotension using homeopathic adrenalin. The results were very promising with complete recovery of sympathetic function, higher heart rate, values of blood pressure and lack of symptoms.

\section{P26}

The role of age and physical training on the baroreflex sensitivity

Melik $Z^{l}$, Cankar $K^{1}$, Strucl $M^{l}$

${ }^{1}$ Institute of Physiology, Medical faculty, University of Ljubljana, Ljubljana, Slovenia 
Aim: Baroreflex sensitivity (BRS) is essential for the short-term control of arterial blood pressure. During aging sensitivity decreases and arterial pressure rises. The aim of present research was to determine how physical training affects BRS in aged individuals.

Methods: Heart rate and arterial blood pressure were monitored in young (20-25 years) healthy trained $(\mathrm{N}=11)$ and sedentary subjects $(\mathrm{N}=11)$ and in elderly (45-55 years) healthy trained $(\mathrm{N}=12)$ and sedentary subjects $(\mathrm{N}=11)$. BRS was defined by the sequence method and spectral technique using arterial pressure oscillations and $\mathrm{R}-\mathrm{R}$ interval changes at rest. On the basis of the arterial pressure change, provoked by the Valsalva manoeuvre, the latency of baroreflex response was determined.

Results: In the group of the elderly untrained men high frequency (alfa HF) component of the BRS was statistically significantly lower $(10.91 .4 \mathrm{~ms} / \mathrm{mmHg}$ ) when compared to the group of the young untrained men $(25.73 .1 \mathrm{~ms} / \mathrm{mmHg})$ (Bonferroni's test, $\mathrm{p}<0.05)$. Decreased BRS in the elderly untrained men $(10.3$ $1.2 \mathrm{~ms} / \mathrm{mmHg}$ ) when contrasted to the young untrained men $(21.0-2.4 \mathrm{~ms} / \mathrm{mmHg}) \quad(\mathrm{p}<0.05)$ was also confirmed by the sequence method. Defining the latency of baroreflex response with the Valsalva manoeuvre proved to be an equally useful indicator for evaluating baroreflex sensitivity. The group of the elderly untrained men showed prolonged delay in the heart rate fall following the arterial blood pressure rise (18.6-2.4 ms) in comparison to the young untrained men $(11.8-1.0 \mathrm{~ms})(\mathrm{p}<0.05)$. The physically active elderly participants did not differ from the young in BRS or in baroreflex response delay.

Conclusions: The research confirmed that physical training improve BRS and baroreflex delay in the group of healthy elder men. 